\title{
Attack Interference in Non-Collaborative Scenarios for Security Protocol Analysis [Extended Version]
}

\author{
M. Camilla Fiazza, Michele Peroli and Luca Viganò
}

July 3, 2018

\begin{abstract}
In security protocol analysis, the traditional choice to consider a single DolevYao attacker is supported by the fact that models with multiple collaborating DolevYao attackers have been shown to be reducible to models with one Dolev-Yao attacker. In this paper, we take a fundamentally different approach and investigate the case of multiple non-collaborating attackers. After formalizing the framework for multi-attacker scenarios, we show with a case study that concurrent competitive attacks can interfere with each other. We then present a new strategy to defend security protocols, based on active exploitation of attack interference. The paper can be seen as providing two proof-of-concept results: (i) it is possible to exploit interference to mitigate protocol vulnerabilities, thus providing a form of protection to protocols; (ii) the search for defense strategies requires scenarios with at least two attackers.
\end{abstract}

\section{Introduction}

\subsection{Context and motivations}

The typical attacker model adopted in security protocol analysis is the one of [16]: the Dolev-Yao (DY) attacker can compose, send and intercept messages at will, but, following the perfect cryptography assumption, he cannot break cryptography. The DY attacker is thus in complete control of the network - in fact, he is often formalized as being the network itself - and, with respect to network abilities, he is actually stronger than any attacker that can be implemented in real-life situations. Hence, if a protocol is proved to be secure under the DY attacker, it will also withstand attacks carried out by less powerful attackers; aside from deviations from the specification introduced in the implementation phase, the protocol can thus be safely employed in real-life networks, at least in principle.

Alternative attacker models have also been considered. On the one hand, computational models for protocol analysis consider attackers who can indeed break cryptography, as opposed to the symbolic models where cryptography is perfect (as we will assume in this paper). See, for instance, [1] for a survey of models and proofs of protocol security, and [6] for a protocol-security hierarchy in which protocols are classified by their relative strength against different forms of attacker compromise.

On the other hand, different symbolic models have been recently proposed that consider multiple attackers instead of following the usual practice to consider a single DY 
attacker, a choice that is supported by the fact that models with multiple collaborating DY attackers have been shown to be reducible to models with one DY attacker (see, e.g., [10] for a detailed proof, as well as [4, 14, 21] for general results on the reduction of the number of agents to be considered). For instance, [5, 20] extend the DY model to account for network topology, transmission delays, and node positions in the analysis of real-world security protocols, in particular for wireless networks. This results in a distributed attacker, or actually multiple distributed attackers, with restricted, but more realistic, communication capabilities than those of the standard DY attacker.

Multiple attackers are also considered in the models of [2, 3, 7, 8], where each protocol participant is allowed to behave maliciously and intercept and forge messages. In fact, each agent may behave as a DY attacker, without colluding nor sharing knowledge with anyone else. The analysis of security protocols under this multi-attacker model allows one to consider scenarios of agents competing with each other for personal profit. Agents in this model may also carry out retaliation attacks, where an attack is followed by a counterattack, and anticipation attacks, where an agent's attack is anticipated, before its termination, by another attack by some other agent.

The features of the models of [5, 20] and of [2, 3, 7, 8] rule out the applicability of the $n$-to- 1 reducibility result for the DY attacker, as the attackers do not necessarily collaborate, and might actually possess different knowledge to launch their attacks. They might even attack each other. In fact, retaliation and anticipation allow protocols to cope with their own vulnerabilities, rather than eradicating them. This is possible because agents are capable of doing more than just executing the steps prescribed by a protocol: they can decide to anticipate an attack, or to counter-attack by acting even after the end of a protocol run (in which they have been attacked). Still, retaliation may nevertheless be too weak as honest agents can retaliate only after an attack has succeeded, and cannot defend the protocol during the attack itself.

\subsection{Contributions}

In this paper, we take a fundamentally different approach: we show that multiple noncollaborating DY attackers may interfere with each other in such a manner that it is possible to exploit interference to mitigate protocol vulnerabilities, thus providing a form of protection to flawed protocols.

To investigate the non-cooperation between attackers, we propose a (protocol-independent) model in which: (i) a protocol is run in the presence of multiple attackers, and (ii) attackers potentially have different capabilities, different knowledge and can interfere with each other. This, ultimately, allows us to create a benign attacker for the system defense: agents can rely on a network guardian, an ad-hoc agent whose task is diminishing the frequency with which dishonest agents can succeed in attacking vulnerable protocols. This methodology moves the focus from an attack-based view of security to a defense-based view.

In other words, in the approach we propose, instead of looking for attacks and reacting to the existence of one by redesigning the vulnerable protocol, we look for strategies for defending against existing known attacks. We would be performing protocol analysis to identify possible defenses, rather than attacks.

We proceed as follows. In Section 2, we formalize models for the network and the agents, including, in particular, agent attitude, goals, and disposition. We then consider in Section 3] a vulnerable protocol from [9] as a case study and focus on the interactions between attack procedures that cannot be observed in classical settings. In Section 4 we explain how interference between attacks leads to a methodology that can be used 
for defending weak (vulnerable) protocols against attacks. In Section 5, we conclude by discussing our approach and current and future work. Appendix A provides additional details about the case study; a second case study is explored in appendix B.

\section{System models: network, agents, attitude}

\subsection{Goals of modeling and approach}

Network models for security protocol analysis typically either replace the communication channel with a single attacker or build dedicated channels for each attacker (e.g. [4, 10, 15, 17, 21]). Traditional modeling strategies are not adequate to describe the non-collaborative scenario under consideration. The main shortcoming is the fact that the ability to spy the communication on a particular channel is hard-wired in the network model and may depend critically on network topology or attacker identity; the result is that an information-sharing mechanism (or a partial prohibition for it) is structurally encoded in the network. We would like, instead, to (i) abstract from positional advantages and focus solely on how attackers interfere by attacking; (ii) treat information-sharing (also as a result of spying) as a strategic choice of the agents.

For simplicity, in this paper we restrict our attention to two non-collaborative attackers $\left(E_{1}\right.$ and $\left.E_{2}\right)$, in addition to the two honest agents $A$ and $B$ and a trusted thirdparty server $S$, whose presence is required by the protocol under consideration. In the following, let Eves $=\left\{E_{1}, E_{2}\right\}$ be the set of attackers and Agents $=\left\{A, B, E_{1}, E_{2}\right\}$ the set of all network agents (honest and dishonest, server excluded). Let $X, Y, Z$ and $W$ be variables varying in Agents and $E$ a variable in Eves; $j$ takes value in $\{1,2\}$, whereas $i \in \mathbb{N}$ is reserved for indexing states.

We are aware that, in situations with more than two (dis)honest agents, further types of interactions can arise; however, a full comprehension of the interactions depends on building a clear picture of interference. Such a picture necessarily starts with the elementary interaction between two attackers.

In order to focus on the raw interference between two attackers, both directing their attack towards the same target, it is important for all attackers to have access to the same view of what is taking place with honest agents and possibly different views of what is taking place with the other attacker(s). If attackers do not all have the same information, it is possible to conceive of strategies in which some attackers can be mislead by others on purpose.

If the knowledge 1 available to an attacker affects his view of the system, attacker capabilities and effectiveness can be diversified, without needing to construct asymmetric attackers or hardwire constraints that may hold for some attackers and not for others. We find it relevant that a network model for non-collaborative scenarios besides reflecting this stance - also support a form of competition for access to messages, especially if attacks rely on erasing messages.

If it is possible in principle to actively interfere with an attack, it should be possible to do so even if all attackers have the same knowledge. However, differentiating attackers with respect to their understanding of the situation - in particular with respect to awareness of other attackers - may bring into focus the conditions, if any, that allow an attacker to interfere with another without being interfered with.

\footnotetext{
${ }^{1}$ Note that we do not attach any epistemic interpretation to the knowledge we consider in this paper we simply consider the information initially available to the agents, together with the information they acquire during protocol executions.
} 
We diversify the activity of our attackers by admitting that attackers may choose to selectively ignore some messages, on the basis of the sender's and receiver's identifiers. This choice reflects actual situations in which attackers pay attention to only a subset of the traffic through a network, focusing on the activity of some agents of interest. Regardless of whether this selection is caused by computational constraints or by actual interest, real attackers filter messages on the basis of the sender's or receiver's identity. In the following, we will use the set Attend $E$ to model the agents to which attacker $E$ is attentive; the predicate ofInterest $_{E}(X)$ (see Table 1) models the decisional process of attacker $E$ as he considers whether he wishes to augment Attend $_{E}$ with $X$, i.e. ofInterest $_{E}(X)$ implies that $X$ is added into Attend $_{E}$.

Honest agents are interested in security properties (such as authentication or secrecy) being upheld through the use of protocols. Dishonest agents, on the other hand, are interested in changing or negating such properties.

The characteristic feature of the attackers we consider is their attitude. In particular, in the case study that we consider in the next section, dishonest agents wish to attack the security protocol and are ready, should they encounter unforeseen interference, to take countermeasures with respect to the interference as well. In a sense, each attacker is exclusively focused on attacking the protocol and becomes aware of other attackers through their effect on his success.

Our target is capturing the behavior of equal-opportunity dishonest agents that do not cooperate in the classical sense. By equal-opportunity attackers we mean agents that have the same attack power and that differ with respect to the information content of their knowledge bases. Such differentiation arises out of attentional choices and not out of intrinsic constraints. Strategic and attitude considerations should not be derivable explicitly from the attacker model — rather, they should configure it.

The driving hypothesis of our work is that studying non-collaboration requires a complex notion of attacker, whose full specification involves attentional choices, decisional processes pertaining to the network environment and to other agents, cooperationrelated choices and decisional processes pertaining to the attack strategy. To support this type of attacker, we extend the usual notions of protocol and role by introducing a control - a mechanism to regulate the execution of the steps prescribed by the attack trace in accordance with the attacker's strategy. In our model, honest agents perform a controlled execution of the protocol as well, so as to support in-protocol detection of attacks. Honest agents behave according to the protocol's prescription, expect things to go exactly in accordance with the protocol and interpret deviations in terms of the activity of dishonest agents.

\subsection{Agent model}

Agent knowledge is characterized in terms of a proprietary dataset. To each $X$ in Agents, we associate the dataset $D_{X}$, which we assume to be monotonically nondecreasing. Our agents, in particular dishonest agents, collect information but do not forget it. When it is important to highlight that the dataset is to be considered at a particular moment, we will use $D_{X}^{i}$ instead.

The network net is also formalized through a dataset, which is named $D_{\text {net }}$ and indexed in the same manner as $D_{X}^{i}$. A dataset is a simple network model that can be configured to support complex attackers; we believe it can successfully meet all of our modeling requirements for non-collaboration. We postpone to Section 2.3 the discussion of how datasets evolve and how indexing and evolution are related to actions and message transmission. 


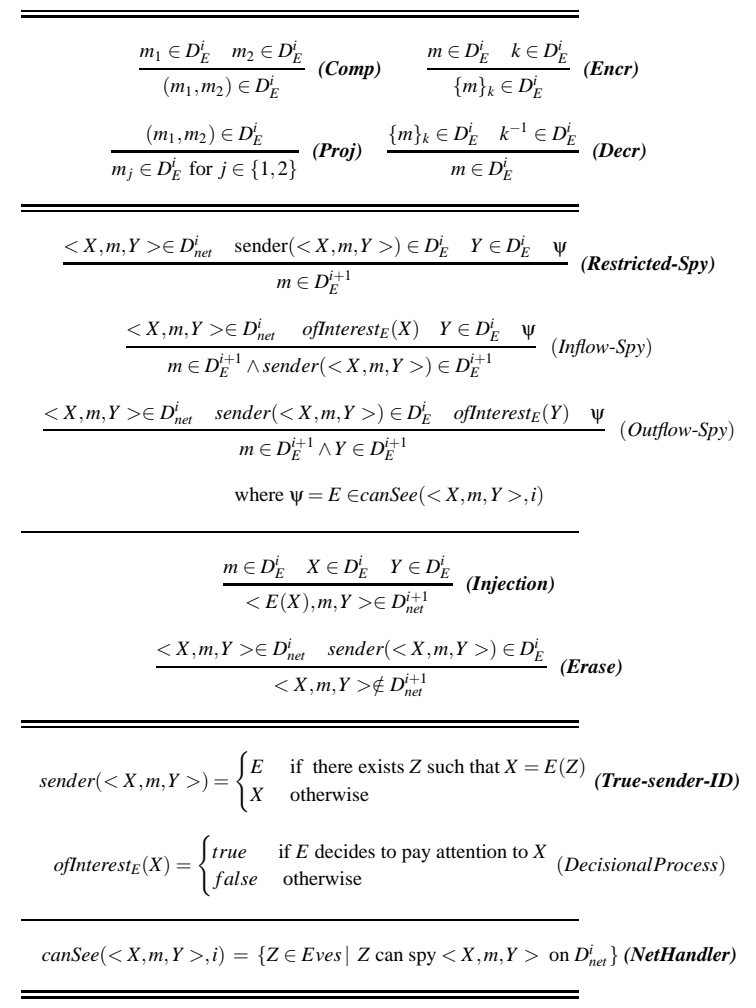

Table 1: Dolev-Yao attacker model for non-collaborative scenarios: internal operations (synthesis and analysis of messages), network operations (spy, inject, erase) and system configuration (True-Sender-ID, DecisionalProcess, NetHandler). NetHandler describes the set of attackers who are allowed to spy by applying one of the spy rules. We omit the usual rules for conjunction. The rules employed in the case study are marked in boldface.

We adapt the notion of DY attacker [16] to capture a non-collaborative scenario. We show in Table1 how one such attacker is formalized within our model, writing rules for attacker $E$ with respect to the knowledge base $D_{E}$ and the network model $D_{n e t}$. Let us specify that the rules in Table 1 are transition rules, rather than deduction rules. Taken altogether, they construct a transition system - which describes a computation by describing the states that are upheld as a result of the transition. We do not intend to carry out in this paper logical inference to identify defenses against attacks; rather, we recognize in the system's evolution what in our eyes corresponds to a defense.

Attackers are legitimate network agents that can send and receive messages, derive new messages by analyzing (e.g. decomposing) known messages, obtain messages transiting on the network (spy) and remove them so that they do not reach their intended receiver (erase). Attackers can also partially impersonate other agents, by injecting messages under a false identity; we represent impersonification with the notation $E(X)$, where $E$ is the impersonator and $X$ is the identifier of the impersonated agent. This set of abilities describes agents who have control over almost all facets of a communication; their characteristic limitation is that they cannot violate cryptography (we assume perfect cryptography). Note that further rules could be added in Table1 for other forms of encryption, digital signatures, hashing, creation of nonces and other 
fresh data, and so on.

The most significant feature concerns spying, represented through three rules. For conceptual clarity, we explicitly pair an erase-rule with the injection-rule, to emphasize that an attacker can modify messages (by erasing them and injecting a substitute) or send messages under a false identity (partial impersonification). Our attackers can employ three different spy rules, adapted to formalize the fact that attackers do not pay attention to all of the traffic on the network. The spy rules rely on an interpretation for "send" that is modified with respect to the denotational semantics in [11], to reflect the attentional focus of attackers. The default spy is the Restricted-Spy: only the messages involving known agents in both sender and receiver roles, regardless of hypotheses on their honesty, become part of the attacker's dataset. Note that in our model what matters is the actual sender and not the declared sender (True-Sender-ID). This mechanism prevents total impersonification and allows filtering messages on the basis of the agent's attentional choices.

The attentional filter we use is meant as a choice of the agents and not as a constraint to which they are subject; therefore, it must be possible to expand the set of agents of interest. This role is fulfilled by the two exploratory spy rules in Table1, Inflow-Spy and Outflow-Spy. Attackers have the option of accepting or rejecting the newly discovered identifier $X$, on the basis of the predicate $\operatorname{ofInterest}_{E}(X)$, which models the decisional process for attention.

Note that an attacker cannot apply any of the spy rules to obtain the message $m$ without knowing the identifier of at least one between $m$ 's sender and $m$ 's intended receiver. By not providing a "generalized spy" rule to waive this requirement, we ensure that $\left(D_{E}^{0} \cap\right.$ Agents $\left.=\emptyset\right)$ implies that for all $i,\left(D_{E}^{i} \cap\right.$ Agents $\left.=\emptyset\right)$. Although $E$ can augment its knowledge base $D_{E}$ indefinitely — through internal message generation and the synthesis rules Comp and Encr -, E's network activity is in fact null. One such $E$ is a dummy attacker, whose usefulness becomes apparent when considering that proof of reductions for non-collaboration can involve progressively migrating identifiers from an attacker's dataset, until the attacker himself reduces to the dummy attacker.

An attacker's dataset $D_{E}$ consists of (i) messages that have transited through the network and that have been successfully received, analyzed or spied and (ii) identifiers of the agents to whom the attacker is attentive. The set Attend $d_{E}$ of identifiers of interest to $E$ is further partitioned into three sets: the set $H_{E}$ of agents believed 7 to be honest, the set $A_{E}$ of agents believed to be attackers, and the set $U_{E}$ of agents whose attitude is unknown in $E$ 's eyes. Note that differently from $D_{n e t}$, agent datasets do not contain triplets ( $\langle$ sender-ID, message, receiver-ID $\rangle$ ), but only messages or identifiers.

Once a new identifier $X$ enters the knowledge base of attacker $E, E$ establishes a belief about the honesty of $X$ and places the identifier in one of the sets $H_{E}, A_{E}$ or $U_{E}$. We do not enter details on how the agents initially build their knowledge base and establish their belief about the attitude of other known agents. In fact, this classification is meant to be dynamic. Agents are on the watch for suspicious messages, which may indicate that an attack is ongoing or may reveal that a certain agent is dishonest. Dynamically adapting their beliefs about the honesty of other agents allows the agents to gather important information during single protocol runs. The agents we wish to consider are smart: they always employ the available strategic information.

Attackers do not have automatic access to triplets that relate sender, message and receiver. They must infer key pieces of information on the basis of the identifiers of the agents to which they are attentive, and attempt to relate the identifiers to the messages

\footnotetext{
${ }^{2}$ We do not attach any doxastic interpretation to the beliefs we consider in this paper.
} 
they spy. Inference is easier if attackers use only the Restricted-Spy rule and keep the set of known agents small. The difficulty of inference rises with the number of attackers in the set AttendE.

\subsection{Network model}

All the operations that can change the state of the network dataset $D_{\text {net }}$ (send, receive, inject and erase) are termed actions, whereas we consider spy simply as an operation: although it requires interacting with the network, it does not change its state. Messages in transit are inserted in the network dataset $D_{\text {net }}$, where attackers can spy them before they are delivered to their intended receivers. Contextually to delivery, the message is removed from the dataset. Messages transit on the network dataset in the form of triplets of the type $\langle$ sender-ID, message, receiver-ID $\rangle$. As a consequence of message delivery or deletion, $D_{\text {net }}$ is non-monotonic by construction.

The sequence of actions that takes place during a protocol run is enumerated and used to index the evolution of the network dataset $D_{\text {net }}$; the index of $D_{\text {net }}^{i}$ is shared with all the proprietary datasets $D_{X}^{i}$, whose states are synchronized accordingly. $D_{\text {net }}^{i}$ is the state of the network dataset after the $i$-th action.

Customarily, evolutions are indexed per transition (per rule application), rather than per action. Our chosen indexing strategy reflects three needs: (1) allowing agents to fully analyze newly acquired messages without having to keep track of the number of internal operations performed; (2) supporting a form of competition between attackers for access to the network; (3) supporting a form of concurrence.

Ideally, all attackers act concurrently. However, the state transitions for the network must be well-defined at all times, even if attackers try to perform conflicting actions, such as spying and deleting the same message in transit. To impose a measure of order, we introduce a network handler, whose task is to regulate the selection of the next action and implement the dependencies between selected action and knowledge available to each attacker; through the network handler, it is also possible to keep the system evolution in accordance with additional constraints, modeling for example information sharing within specific subsets of agents and network topology.

As soon as the state of the network changes (e.g. as a result of inject or send), the network handler passes the new triplet to each attacker, who then simulates spying and decides on whether to request erasing the message or injecting a new one as a consequence, in accordance with his strategy. The network handler interprets the application of the spy-rules, the inject-rule and the erase-rule as requests and selects the next action from the set of requests. Message deletion, when requested by any attacker, is always successful.

The outcome of the process governed by the network handler is described through the function $\operatorname{canSee}($ ), which returns a subset of Eves, highlighting the identifiers of the attackers who can spy "before" the message is erased from $D_{\text {net }}$. The set of agents described by $\operatorname{canSee}($ ) contains at least the identifier of the attacker whose erase request was served.

If the network handler does not receive any erase-requests, all attentive attackers can acquire the message. If one or more erase-requests are present, the network handler erases the message and confirms success in spying only for a subset of attentive attackers. If an attacker is not in canSee(), the prior (simulated) spy is subject to rollback, along with all internal operations that have occurred since the last confirmed action. If no requests are received from attackers, the network handler oversees message delivery or selects actions requested by honest agents. 


\begin{tabular}{cl}
\hline$(i+1)^{\text {th }}$ action & Formalization \\
\hline$X \rightarrow Y: m$ & $m \in D_{X}^{i}$ and $Y \in D_{X}^{i}$ \\
& $<X, m, Y>\in D_{\text {net }}^{i+1} \quad$ and $\quad<X, m, Y>\notin D_{\text {net }}^{i+2}$ \\
& $m \notin D_{W}^{i+2}$, where $W \notin$ canSee $(<X, m, Y>, i+1)$ \\
& $m \in D_{Y}^{i+2}$ \\
\hline$E(X) \rightarrow Y: m$ & $m \in D_{E}^{i}$ and $X \in D_{E}^{i} \quad$ and $\quad Y \in D_{E}^{i}$ \\
& $<E(X), m, Y>\in D_{\text {net }}^{i+1} \quad$ and $\quad<E(X), m, Y>\notin D_{\text {net }}^{i+2}$ \\
& $m \notin D_{W}^{i+2}$, where $W \notin$ canSee $(<X, m, Y>, i+I)$ \\
& $m \in D_{Y}^{i+2}$ \\
& $m \in D_{X}^{i}$ and $Y \in D_{X}^{i}$ \\
& $<X, m, Y>\in D_{\text {net }}^{i+1} \quad$ and $\quad<X, m, Y>\notin D_{\text {net }}^{i+2}$ \\
& $m \in D_{W}^{i+2}$, where $W \in I$ and $I \subseteq$ canSee $(<X, m, Y>, i+1)$ \\
\hline$X \rightarrow E_{I}(Y): m$ &
\end{tabular}

Table 2: Representation of operations in Alice\&Bob notation.

Although the formulation of $\operatorname{canSee}()$ in terms of access time is intuitive, the reason why we favor this mechanism is that time-dependent accessibility is not the only situation it can model. The function can be instantiated to model strategic decision-making and information-sharing, or to capture a particular network topology. In realistic attack scenarios, knowledge of a message that has been erased may depend more on cooperation and information-sharing than on timing. For example, if $E_{j}$ is sharing information with $E_{k}$ (but not viceversa), whenever $E_{j}$ 's erase requests are served $E_{k}$ is automatically in $\operatorname{canSee}()$.

The network handler is not an intelligent agent. Specifying its behavior and instantiating the function canSee() corresponds to configuring the particular network environment in which the agents are immersed (i.e. $\operatorname{canSee}()$ is a configurable parameter of our model).

As a result on the network handler and of our chosen indexing strategy, several internal operations can occur in a proprietary dataset between consecutive states, whereas only a single action separates consecutive states of the network dataset. Attackers determine the next state of the network dataset with priority with respect to the actions of honest agents.

In Table 2, we formalize within our model operations in the Alice\&Bob notation used in Section 3, we write $E_{I}(Y)$ to denote the subset of Eves who spy message $m$ addressed to $Y$, at least one of which has requested $m$ to be erased.

With reference to Table 2, note that the $(i+1)^{\text {th }}$ action is requested when the state of the network is $D_{n e t}^{i}$ and agent datasets are $D_{X}^{i}$; thus, the sender $X$ must already know in $D_{X}^{i}$ both the message $m$ and the identifier of the intended recipient $Y$. The message correctly transits on $D_{\text {net }}^{i+1}$, immediately after being sent. The $(\mathrm{i}+2)$ th action is either receive (first two cases) or erase (last case). the availability of $m$ to attackers is conclusively decided after the network handler selects the $(i+2)$ th action, and thus pertains to $D_{W}^{i+2}$.

\subsection{Attacker goals and agent disposition}

The notion of cooperation between agents can be viewed from at least two perspectives of interest: sharing of information and sharing of success. The notion of attacker cooperation classically employed in protocol analysis encompasses both aspects, as it states the first while assuming that the second holds. 


\begin{tabular}{|c|c|c|c|c|c|}
\hline \multicolumn{3}{|r|}{ BME } & \multicolumn{3}{|c|}{ Classical Attack } \\
\hline $\begin{array}{l}(1) \\
\text { (2) } \\
\text { (3) }\end{array}$ & $\begin{array}{l}A \rightarrow S \\
S \rightarrow A \\
A \rightarrow B\end{array}$ & $\begin{array}{l}: A, B \\
:\left\{\left|k_{A B}\right|\right\}_{k_{A S}},\left\{\left|k_{A B}\right|\right\}_{k_{B S}} \\
:\left\{\left\{k_{A B} \mid\right\}_{k_{B S}}\right.\end{array}$ & $\begin{array}{l}(1) \\
\left(1^{\prime}\right) \\
(2) \\
(3)\end{array}$ & $\begin{array}{l}A \rightarrow E(S) \\
E(A) \rightarrow S \\
S \rightarrow A \\
A \rightarrow E(B)\end{array}$ & $\begin{array}{l}: A, B \\
: A, E \\
:\left\{\left|k_{A E}\right|\right\}_{k_{A S}},\left\{\left|k_{A E}\right|\right\}_{k_{E S}} \\
:\left\{\left|k_{A E}\right|\right\}_{k_{E S}}\end{array}$ \\
\hline
\end{tabular}

Table 3: The Boyd-Mathuria Example protocol and a masquerading attack against it.

In this paper, we examine attackers that exhibit, with respect to cooperation, the behavior we call complete non-collaboration: agents voluntarily abstain from sharing information and do not consider their goals as met if they do not succeed in attacking. The disposition of attacker $E_{1}$ towards $E_{2}$ belongs to one of the following basic classes: active collaboration, passive collaboration, competition and conflict3. The focus of this paper is on competition - a situation in which the goal is successfully attacking the protocol, regardless of the disposition of other agents. From the perspective of a competitive attacker, other attackers are not of interest per se: they are relevant factors because they are sources of interference. If some interference is detected while carrying out an attack, a competitive attacker will take countermeasures, attempting to negate potentially adverse effects.

Sets of agents that are homogeneous with respect to disposition can be used to define scenarios of interest. In the case study below, we explore a simple characteristic scenario composed of two competitive attackers; we aim to bring into focus the mechanisms by which two attackers can affect each other's success.

\section{A case study: the Boyd-Mathuria Example}

A dishonest agent, aware that other independent attackers may be active on the network, will seek to devise suitable novel attacks, so as to grant himself an edge on unsuspecting competitors. As the mechanics of interaction and interference between attackers have not been exhaustively studied in literature yet, it is not known a priori how to systematically derive an attack behavior of this type.

In the following case study, we start from a simple protocol for which a vulnerability is known; we devise for the known ("classical") attack a variant that explicitly considers the possibility of ongoing independent attacks. We describe a possible reasoning for a competitive attacker in the context of the protocol's main features. Due to space limitations, we give additional details about the case study in the appendix.

The protocol we consider as a case study is a key transport protocol described as an example in [9]; we name it as the Boyd-Mathuria Example (BME), and present it in Table 3 together with a classical attack against it. BME relies on the existence of a trusted third-party server $S$ to generate a session key $k_{A B}$ for agents $A$ and $B$, where each agent $X$ is assumed to share a symmetric secret key $k_{X S}$ with $S$.

$A$ is subject to a masquerading attack in which, at the end of a run of BME, $A$ thinks that he shares a session key with the honest agent $B$, while in fact he shares it with the attacker $E$. Subsequent communication from $A$ addressed to $B$ is seen by $E$ through the spy-rule and removed with an erase request: $E$ has successfully taken $B$ 's place. This

\footnotetext{
${ }^{3}$ In active and passive collaboration there is a common goal to be pursued; the difference lies in choosing a strategy that helps another vs. choosing a strategy that does not hinder another. In conflict scenarios, the primary focus of interest is the attackers, rather than the protocol.
} 
attack prevents $B$ from receiving any communication from $A$. Should the two agents have prior agreement that such a communication was to take place, $B$ is in the position of detecting that something has gone wrong. $E$ can prevent detection by staging a dual man-in-the-middle attack.

If more than one attacker is active during a given protocol run, simultaneous execution of the classical attack could lead to $A$ receiving multiple session keys as a response to his (single) request to the server. This situation clearly indicates to $A$ that an attack is ongoing. A competitive attacker $E_{1}$, wishing to prevent this situation from occurring, could try removing from the network all the responses from $S$ to $A$ that do not pertain to his own request. However, the characteristics of the (non-redundant) cryptographic methods employed here do not allow distinguishing $M_{1}=\left(\left\{\left|k_{A E_{1}}\right|\right\}_{k_{A S}},\left\{\left|k_{A E_{1}}\right|\right\}_{k_{E_{1} S} S}\right)$ (to let through) from $M_{2}=\left(\left\{\left|k_{A E_{2}}\right|\right\}_{k_{A S}},\left\{\left|k_{A E_{2}}\right|\right\}_{k_{E_{2} S}}\right)$ (to block). $E_{1}$ can recognize the format of $M_{1}$ and $M_{2}$ and can successfully decrypt $M_{1}$ to recover $k_{A E_{1}}$; by decrypting $M_{2}$ with the key $k_{E_{1} S}, E_{1}$ can still recover a value, but different from the previous one. Not knowing $k_{A E_{1}}$ a priori, the attacker is not able to distinguish which of $M_{1}$ and $M_{2}$ contains the answer to his request for a key with $A$.

As a consequence, the attacker $E_{1}$ is not able to know which messages to remove in order to ensure that $A$ accepts $k_{A E_{1}}$ as a session key to communicate with $B$. Competitive attackers cannot rely on step (2) to enforce their attacks at the expense of their competitors; furthermore, the probability of erasing all competing messages (while letting one's own pass) decreases with the number of active attackers. In this situation, it becomes fundamental for a competitive attacker to gain exclusive access to the first message and gain control over the messages that reach $S$, as opposed to the messages coming from $S 4$.

After spying the initiator's opening message, a competitive attacker $E_{1}$ will therefore attempt to mount the classical attack, while keeping watch for other messages that may be interpreted as attack traces. Any transiting message of the type $\left(A, E_{m}\right)$ for which $E_{m} \in A_{E_{1}}$ is interpreted as another active attack; $E_{1}$ counters by requesting that the message be erased. If $E_{m}$ is in $H_{E_{1}}$, the message may be understood either as a message from $A$ - who would be initiating a parallel session of the protocol to obtain a second session key - or as an indication that $E_{m}$ has been incorrectly labeled as honest. In the first case, $E_{1}$ will let the message through, as he has chosen to target specifically the session key for the communication between $A$ and $B$; in the second case, he will protect his attack by erasing the message. If $E_{m}$ is in $U_{E_{1}}, E_{1}$ can choose to either play conservatively and hypothesize the dishonesty of $E_{m}$ or let the message through and interpret $E_{m}$ as the culprit in case the current attack fails.

$\mathrm{BME}$ is such that at most one attacker $E_{d}$ can successfully mislead $A$ into accepting the key $k_{A E_{d}}$ as a session key to communicate with $B$. Therefore, a successful attack automatically entails exclusivity of success. An attack is successful if it goes undetected by the initiator $A$. Our honest agents are intelligent and they make use of all information available to perform in-protocol detection of attacks. With respect to BME, a clear indication for $A$ consists in receiving multiple responses from $S$ after a single session key request; if $A$ receives multiple responses, he concludes that there has been a security violation and thus does not employ any of the keys so received in his later communications with $B$ - choosing to try a fresh run of the protocol instead. From the attackers' perspective, an ongoing attack can be detected by observing a message

\footnotetext{
${ }^{4}$ Of course, $E_{1}$ could guess which message(s) to erase, but he would have the added difficulty of having to decide whether to let the first message pass without knowing how many other messages will transit, if any at all, and how many session keys were requested by $A$ (as opposed to by his competitor(s)).
} 
of the type $(A, X)$ transiting on the network; however, the attack trace is ambiguous to spying attackers and has to be interpreted on the basis of current beliefs concerning the honesty of $X$. A last feature of interest is that BME is rather friendly for attacker labeling. Decisional processes can rely on at least some conclusive information on the identity of the agents involved, because identifiers transit in the clear; attackers would have to infer them otherwise.

We examine the outcome of attacks carried out in a non-collaborative environment in six cases, corresponding to different conditions of knowledge and belief for $E_{1}$ and $E_{2}$. Cases and attack traces are summarized in Table 4 In order to completely specify agent behavior, we posit the following:

1. If an attacker $E$ spies $\left(A, E_{m}\right)$ with $E_{m} \in H_{E}$ or $E_{m} \in U_{E}$, he will not request that the message be erased. In the latter case, if $E$ 's attack fails, $E_{m}$ is immediately placed in $A_{E}$.

2. Both $E_{1}$ and $E_{2}$ spy the opening message and are interested in attacking the current protocol run; this allows us to leave aside the trivial cases in which only one attacker is active for a given protocol run.

3. Due to space constraints, we detail only the cases in which canSee for step (3) yields $\left\{E_{1}, E_{2}\right\}$. Cases in which only one of the attackers can access $A$ 's response can be found in appendix $\mathrm{A}$

Case 1: $E_{1}$ and $E_{2}$ know each other as honest.

$E_{1}$ and $E_{2}$ know each other's identifiers (i.e. they are paying attention to each other: $E_{1} \in D_{E_{2}}$ and $\left.E_{2} \in D_{E_{1}}\right)$, but they are both mistaken in that they have labeled the other as honest $\left(E_{1} \in H_{E_{2}}\right.$ and $\left.E_{2} \in H_{E_{1}}\right) . E_{1}$ and $E_{2}$ are unaware of active competitors and mount the classical attack in steps $\left(1_{1}\right)$ and $\left(1_{2}\right)$. When the attackers spy two requests to the server transiting on the network, they both believe that $A$ wishes to request keys with the honest agents B and $E_{j}$.

(1.T1): $S$ sends two messages before A can address a message to $B$. With the messages in steps $\left(2_{1}\right)$ and $\left(2_{2}\right), A$ receives two keys instead of the single key requested. $A$ now knows that at least one attacker is active and abandons the protocol without sending a message to $B$. The attackers do not spy the message they were hoping for (timeout) and acquire the certainty that at least another active attacker is around. The attackers can employ ad-hoc strategies to search for the mislabeled or unknown attacker. If the attackers are careful to keep track of the messages $(A, X)$ pertaining to a given session, they can make informed guesses as to whom, amongst the known agents, they might have mislabeled.

(1.T2): A receives a reply from $S$, answers $B$ and stops listening. A receives the messages he expects and closes the current session before receiving the second response from $S . E_{1}$ is successful in his attack, whereas $E_{2}$ believes that he has succeeded when he has, in fact, decrypted the wrong key. None of the agents have an opportunity for detection.

(1.T3): A receives a reply from $S$, answers $B$ and keeps listening. $A$ replies with the message in step (3), resulting in both $E_{1}$ and $E_{2}$ believing that they have succeeded. However, after receiving $\left(2_{2}\right), A$ detects the attack and abstains from employing $k_{A E_{1}}$ in his future communications with $B$. Thus, even if for different reasons, both attackers in fact fail. Furthermore, they both continue to hold their mistaken belief that the other attacker is in fact honest.

Case 2: $E_{1}$ and $E_{2}$ know each other as attackers. 


\begin{tabular}{|c|c|c|c|c|c|}
\hline \multicolumn{3}{|c|}{ T1: cases $1,3,4,6$} & \multicolumn{3}{|c|}{$T 2$ and [T3]: cases $1,3,4,6$} \\
\hline $\begin{array}{r}(1) \\
\downarrow\left(1_{1}\right) \\
\uparrow\left(1_{2}\right) \\
\left(2_{1}\right) \\
\left(2_{2}\right)\end{array}$ & $\begin{array}{l}A \rightarrow E_{1,2}(S) \\
E_{1}(A) \rightarrow S \\
E_{2}(A) \rightarrow S \\
S \rightarrow A \\
S \rightarrow A\end{array}$ & $\begin{array}{l}: A, B \\
: A, E_{1} \\
: A, E_{2} \\
: M_{1} \\
: M_{2}\end{array}$ & $\begin{array}{r}(1) \\
\left.\downarrow(1)_{1}\right) \\
\uparrow\left(1_{2}\right) \\
\left(2_{1}\right) \\
(3) \\
{\left[\left(2_{2}\right)\right.}\end{array}$ & $\begin{array}{l}A \rightarrow E_{1,2}(S) \\
E_{1}(A) \rightarrow S \\
E_{2}(A) \rightarrow S \\
S \rightarrow A \\
A \rightarrow E_{1,2}(B) \\
S \rightarrow A\end{array}$ & $\begin{array}{l}: A, B \\
: A, E_{1} \\
: A, E_{2} \\
: M_{1} \\
:\left\{\left|k_{A E_{1}}\right|\right\}_{k_{E_{1}}} S \\
\left.: M_{2}\right]\end{array}$ \\
\hline \multicolumn{3}{|c|}{ T4: case 2} & \multicolumn{3}{|c|}{ T5: case 5} \\
\hline $\begin{aligned} &(1) \\
& \downarrow\left(1_{1}\right)^{+} \\
& \uparrow\left(1_{2}\right)^{+}\end{aligned}$ & $\begin{array}{l}A \rightarrow E_{1,2}(S) \\
E_{1}(A) \rightarrow E_{2}(S) \\
E_{2}(A) \rightarrow E_{1}(S)\end{array}$ & $\begin{array}{l}: A, B \\
: A, E_{1} \\
: A, E_{2}\end{array}$ & $\begin{array}{l}(1) \\
\downarrow\left(11_{1}\right) \\
\uparrow\left(1_{2}\right) \\
(2) \\
(3)\end{array}$ & $\begin{array}{l}A \rightarrow E_{1,2}(S) \\
E_{1}(A) \rightarrow E_{2}(S) \\
E_{2}(A) \rightarrow S \\
S \rightarrow A \\
A \rightarrow E_{1,2}(B)\end{array}$ & $\begin{array}{l}: A, B \\
: A, E_{1} \\
: A, E_{2} \\
: M_{2} \\
:\left\{\left|k_{A E_{2}}\right|\right\}_{k_{E_{2}}} S\end{array}$ \\
\hline
\end{tabular}

Where: $M_{1}=\left\{\left|k_{A E_{1}}\right|\right\}_{k_{A S}},\left\{\left|k_{A E_{1}}\right|\right\}_{k_{E_{1}} S}, \quad M_{2}=\left\{\left|k_{A E_{2}}\right|\right\}_{k_{A S} S},\left\{\left|k_{A E_{2}}\right|\right\}_{k_{2} S}$

Table 4: Traces for non-collaborative attacks against BME. Traces are exhaustive: $E_{1}$ and $E_{2}$ have priority over honest agents and $S$ is honest. Arrows: relative order between $\left(1_{1}\right)$ and $\left(1_{2}\right)$ is irrelevant in determining the outcome.

$E_{1}$ and $E_{2}$ know each other's identifier $\left(E_{1} \in D_{E_{2}}\right.$ and $\left.E_{2} \in D_{E_{1}}\right)$ and have correctly understood that the other is behaving as a dishonest agent $\left(E_{1} \in A_{E_{2}}\right.$ and $\left.E_{2} \in A_{E_{1}}\right)$. Each attacker is aware of the presence of a competitor, which they have correctly labeled. Each attacker is attempting to gain exclusive access to the initial communication towards $S$ and to ensure that only his request reaches $S . E_{1}$ and $E_{2}$ erase each other's request to $S$. Within our model, no attacker can be certain that his message has been received by its intended receiver; the attackers may wish to replay step $\left(1_{1}\right)$ and $\left(1_{2}\right)$ if a message of the type $\left\{\left|k_{A E_{j}}\right|\right\}_{k_{A S}},\left\{\left|k_{A E_{j}}\right|\right\}_{k_{E_{j}}}$ is not spied on the network within a reasonable time. This option is marked with $(\cdot)^{+}$in Table 4 However, the active presence of the competitor ensures that no message reaches $S$. A notices that an anomalous situation is occurring, because his request to the server is not being served in a reasonable time. $A$ interprets the situation as a denial-of-service attack and abandons the protocol.

\section{Case 3: $E_{1}$ and $E_{2}$ are unaware of each other.}

$E_{1}$ and $E_{2}$ are unaware of the other's presence - i.e. they are not paying attention to the other's activity $\left(E_{1} \notin D_{E_{2}}\right.$ and $\left.E_{2} \notin D_{E_{1}}\right)$. Subcases follow closely those described for case 1 above. The only significant difference concerns detection for trace T1: here the attackers must employ exploratory strategies (Inflow-Spy or Outflow-Spy), because they failed to spy an additional message of type $\left(A, E_{m}\right)$ transiting on the network. The failure to observe such a message is a strong indicator that the competitor's identifier is unknown. In 2-attacker scenarios this is the only legitimate conclusion, whereas with three or more attackers this situation may also arise from the interplay between erase and spy operations.

Case 4: $E_{2}$ knows $E_{1}$ as honest.

Only one out of the two attackers $E_{1}$ and $E_{2}$ is paying attention to the other and knows his identifier. Here we consider $E_{1} \in H_{E_{2}}$ and $E_{2} \notin D_{E_{1}}$. Regardless of the order in which steps $\left(1_{1}\right)$ and $\left(1_{2}\right)$ occur, the attacker in disadvantage $E_{1}$ does not spy the message at step $\left(1_{2}\right) ; E_{2}$ does spy $\left(1_{1}\right)$ but, trusting his judgement on $E_{1}$ 's honesty, does not request it to be erased. As a consequence, similarly to case 1 , the traces follow schemes T1, T2 and T3. Significant differences concern detection in T1: $E_{1}$ detects 
the presence of an unknown attacker, whereas $E_{2}$ learns of a mislabeled or unknown attacker. The successful attackers in traces T2 and T3 are those whose requests to $S$ are served first; knowledge does not affect the outcome.

Case 5: $E_{2}$ knows $E_{1}$ as dishonest.

Only one out of the two attackers $E_{1}$ and $E_{2}$ is paying attention to the other and knows his identifier. Here we consider $E_{1} \in A_{E_{2}}$ and $E_{2} \notin D_{E_{1}}$ Regardless of the order in which steps $\left(1_{1}\right)$ and $\left(1_{2}\right)$ occur, $E_{1}$ does not spy the message at step $\left(1_{2}\right)$ and $E_{2}$ uses a direct attack against the competitor. $E_{2}$ removes $E_{1}$ 's request to the server and remains the only attacker in play, leading $A$ into accepting $k_{A E_{2}}$ as a session key. $E_{1}$ does not have an opportunity to detect the competitor.

Case 6: $E_{2}$ knows $E_{1}$, but he is unsure of $E_{1}$ 's honesty.

Only one out of the two attackers $E_{1}$ and $E_{2}$ is paying attention to the other and knows his identifier. Here we consider $E_{1} \in U_{E_{2}}$ and $E_{2} \notin D_{E_{1}}$. This case reduces to case 4, with the only difference that $E_{2}$ is testing the dishonesty of $E_{1}$, instead of believing his honesty. Whenever $E_{2}$ realizes that he has failed his attack, he adds $E_{1}$ into $A_{E_{2}}$ and deletes it from $U_{E_{2}}$.

\section{General considerations.}

In traces T2 and T3, the winning attacker is the one whose request is served first by $S$. $S$ is an honest agent but it is not constrained to answering requests in the exact order in which they are received. Attackers do not have control over which requests are served first, although this factor determines whether they cannot do better than acquire the wrong key. Attackers realize in-protocol that they have failed only when they cannot spy a response from $A$, i.e. when they do not acquire any keys. Post-protocol detection, on the other hand, can occur also when an attacker with a wrong key attempts to decrypt the later communications addressed by $A$ to $B$.

The case study highlights that, if $A$ keeps the session open for a reasonable time after step (3), he can improve his chances of discovering that the key is compromised. This is a simple strategy that is beneficial and does not depend on the particular protocol. Furthermore, when $A$ receives two answers from $S$ in response to his single request, he now has two keys - at least one of which is shared with an attacker. If honest agents are immersed in a retaliatory framework [7, 8], such keys can be used to identify attackers, to feed them false information or, in general, to launch well-aimed retaliatory attacks.

\section{Defending vulnerable protocols against attacks}

Key exchange protocols are amongst the most used cryptographic protocols. It is a common security practice to establish a secure channel by first exchanging a session key and then using it to authenticate and encrypt the data with symmetric cryptography. The security of all communications occurring during a session rests on the integrity of the key. In this context, it is not important per se that a key has been acquired by an attacker: what matters is whether a compromised key is used. Rather then on preventing the acquisition of a session key from ever occurring, the focus is on detecting that the key has been compromised - so as to prevent an attack from spreading to the entire session traffic.

If a protocol is vulnerable, a single DY attacker will succeed with certainty. How- 


\begin{tabular}{c|cccc}
\hline canSee & Cases & Case 2 & $\begin{array}{c}\text { Case 5: } \\
E \in A_{G}\end{array}$ & $\begin{array}{c}\text { Case 5: } \\
G \in A_{E}\end{array}$ \\
\hline$\{E, G\}$ & $\sim^{+}$ & $\sqrt{ }$ & $\sqrt{ }$ & \\
$\{G\}$ & $\sqrt{ }$ & $\sqrt{ }$ & $\sqrt{ }$ & $\sqrt{ }$ \\
$\{E\}$ & $\sim^{+}$ & $\sqrt{ }$ & $\sqrt{ }$ & \\
\hline
\end{tabular}

Table 5: Effects of introducing a guardian $G$ for BME when attacker $E$ is active. $G$ operates according to the same strategy as the attackers in the case study. $G$ 's active interference results in $A$ detecting attacks always $(\sqrt{ })$, sometimes $(\sim)$, always if $A$ commits to listening after step (3) $\left({ }^{+}\right)$. The guardian is progressively more effective the more his beliefs and knowledge reflect the actual set of attackers. $G$ can be effective even when he is not aware of $E$ 's presence.

ever, if attacks to the same protocol are carried out in a more complex network environment, success is not guaranteed. As shown in the case study, in competitive scenarios with equal-opportunity attackers it is not possible for a given attacker to ensure that an attack is successful under all circumstances. The outcome depends on the strategy and knowledge conditions of all the active agents, on the visibility of erased messages to other attackers $\left(\right.$ canSee $\left.\neq\left\{E_{1}, E_{2}\right\}\right)$ and on the order with which $S$ processes requests. In a sense, the presence of an independent active attacker constrains the success of otherwise sure-fire attacks.

This principle can be exploited to facilitate detection of attacks against vulnerable protocols. Honest agents should not, in principle, be informed of the specific attack trace to which they are vulnerable. Hence, if honest agents can perform detection at all, it has to be on the basis of flags that are independent of the specific attack trace - and, in general, independent also of the protocol in use. Such flags encode local defense criteria and can be as simple as realizing that no answer has arrived within a time considered reasonable or realizing that two (different) answers have been sent in response to a single request.

The basic idea is constructing a network agent that causes protocol-independent flags to be raised - via deliberate interference with ongoing attacks. In addition, one such guardian agent is formally an attacker, and can therefore be configured with knowledge of the attack trace(s). The guardian's task can be formulated as raising protocol-independent flags in correspondence to protocol-dependent indicators.

By using such an ad-hoc competitor as defense, it is possible, in some cases, to allow detection of otherwise-undetectable attacks. If no flag is raised for $A$, the guardian may be the only attacker at work. In this case, no ill-intentioned attacker has successfully concluded an attack; from the standpoint of $A$, actual security is not affected. A guardian is a practical solution even when it is not all-powerful: any attack detected by $A$ thanks to the guardian's active presence is an improvement in security. In Table 5] we show the effects of introducing a guardian $G$ for BME, configured as the attackers in the case study. It is not necessary to demand that the guardian monitor all traffic - which is unrealistic at best; on the other hand, all monitored traffic enjoys partial protection.

Attacks failing are, by themselves, markers that there are other dishonest agents at work; this fact can be used by the guardian $G$ as a basis for further detection, possibly on behalf of honest agents. Then guess-and-test strategies can be used to acquire an understanding of the second attacker's identity; a rudimentary example is the strategy 
used by our attackers for BME when they spy $\left(A, E_{m}\right)$ and $E_{m} \in U$. Across multiple iterations of the attack procedure and under different hypotheses concerning $\left(H_{G}, A_{G}\right.$, $U_{G}$ ), the attacker's identity will eventually be revealed.

In actual scenarios, protocols are implemented through programs in the users' computers. It is very difficult to force users to stop using a protocol as soon as a vulnerability is discovered. The more widespread the protocol, the more difficult it is to ensure that it quickly goes out of use. Two aspects are important: that every user (a) is informed of the new vulnerability and (b) takes action in switching to a secure protocol. Statistics on software upgrades are an unfortunate example of this type of issue.

By designing the user-end software to inform the user of a security failure whenever protocol-independent flags are raised, a guardian can help solve the notification issue as well as raise the likelihood that the user will take action and upgrade. When the weakness in the protocol is understood, it may be a cost-effective investment to design a guardian with an effective interference strategy, so as to facilitate restoring network security.

\section{Conclusions and future work}

The traditional goal of protocol analysis is discovering attacks, to prompt replacing a vulnerable protocol with an improved and more secure one. Reductions are centered on attacks, either to reduce the search space for attacks (e.g. [4, 18, 19]) or to reduce the number of agents (e.g. [4, 14]). In particular, if there exists an attack involving $n$ collaborating attackers, then there exists an "equivalent" attack involving only one. Within this perspective, it is known that $n$-DY attackers equal in attack power a single DY attacker, and that the same can be said of Machiavelli-type attackers [16, 21]. As a result, an exhaustive search for attacks can be performed in a reduced-complexity model.

On the other hand, within our proposed approach the goal of analysis is finding a strategy to defend the system against existing attacks, rather than identifying vulnerabilities to prompt redesigning the protocol. We would be performing protocol analysis to identify possible defenses, rather than attacks.

In the case study, we have shown a counterexample to the statement: "if there exists a defense against an attack in a 2-attacker scenario, then there exists an equivalent defense in a 1-attacker scenario". This statement mirrors the classical result on $n$ to- 1 reducibility and the counterexample shows that exhaustive searches for defenses against attacks cannot be carried out in reduced-complexity settings, as they require at least two attackers.

Having chosen vulnerable protocols, in a single-attacker situation there is no protocol-independent indicator that could be used by honest agents to become aware that security has been compromised. If there is a single attacker, no simple defense is possible and the protocol inevitably fails its security goals. On the other hand, by exploiting an ad-hoc competitor (the guardian) as a defense, in certain conditions we can successfully raise protocol-independent indicators of ongoing attacks and protect the system. Introducing an appropriate guardian procedure as soon as new attacks are discovered can mitigate the consequences of flawed protocols still being in use.

Along the line of work presented in this paper, we have investigated two additional simple protocols as case studies: the Shamir-Rivest-Adleman three-pass protocol, which differs significantly from BME in that success is not necessarily exclusive, and the Beller-Yacobi protocol, which requires interacting with a second honest agent 
to carry out a masquerading attack. The goal of these investigations is to bring into focus how the salient features of each protocol are reflected in the possible mechanisms of interference. The first case study is available as additional material in appendix B. A second topic of interest is evaluating (i) whether the mechanisms of interaction highlighted in two-attacker scenarios are directly portable to situations with more than two non-collaborating attackers, (ii) whether they require ad-hoc generalization and (iii) whether new types of interaction emerge when more than two dishonest agents are active. We are investigating this in more detail, along with a (semi-)automatic implementation of our approach.

\section{Acknowledgements}

The work presented in this paper was partially supported by the FP7-ICT-2007-1 Project no. 216471, AVANTSSAR: Automated Validation of Trust and Security of Serviceoriented Architectures (www.avantssar.eu) and by the FP7-ICT-2009-5 Project no. 257876, "SPaCIoS: Secure Provision and Consumption in the Internet of Services" (www.spacios.eu). We thank Davide Guardini for his constructive comments.

\section{References}

[1] Martín Abadi, Bruno Blanchet, and Hubert Comon-Lundh. Models and proofs of protocol security: A progress report. In Proceedings of 21st International Conference on Computer Aided Verification (CAV'09), LNCS 5643, pages 3549. Springer, 2009.

[2] Wihem Arsac, Giampaolo Bella, Xavier Chantry, and Luca Compagna. Validating Security Protocols under the General Attacker. In Proceedings of the Joint Workshop on Automated Reasoning for Security Protocol Analysis and Issues in the Theory of Security (ARSPA-WITS 2009), LNCS 5511, pages 34-51. Springer, 2009.

[3] Wihem Arsac, Giampaolo Bella, Xavier Chantry, and Luca Compagna. Multiattacker protocol validation. Journal of Automated Reasoning, in press.

[4] David Basin, Carlos Caleiro, Jaime Ramos, and Luca Viganò. Distributed temporal logic for the analysis of security protocol models. , .

[5] David Basin, Srdjan Capkun, Patrick Schaller, and Benedikt Schmidt. Let's get physical: Models and methods for real-world security protocols. In Proceedings of TPHOLs'09, LNCS 5674, pages 1-22. Springer, 2009.

[6] David Basin and Cas Cremers. Modeling and analyzing security in the presence of compromising adversaries. In Proceedings of ESORICS 2010, LNCS 6345, pages 340-356. Springer, 2010.

[7] Giampaolo Bella, Stefano Bistarelli, and Fabio Massacci. A protocol's life after attacks. In Proceedings of 11th International Workshop on Security Protocols, LNCS 3364, pages 3-18. Springer, 2003.

[8] Giampaolo Bella, Stefano Bistarelli, and Fabio Massacci. Retaliation against protocol attacks. Journal of Information Assurance and Security, 3:313-325, 2008. 
[9] Colin Boyd and Anish Mathuria. Protocols for Authentication and Key Establishment. Springer, 2003.

[10] Carlos Caleiro, Luca Viganò, and David Basin. Metareasoning about security protocols using distributed temporal logic. Electronic Notes in Theoretical Computer Science, 125(1):67-89, 2005.

[11] Carlos Caleiro, Luca Viganò, and David Basin. On the semantics of Alice \& Bob specifications of security protocols. Theoretical Computer Science, 367(1-2):88 $-122,2006$.

[12] Ulf Carlsen. Cryptographic protocol flaws. In 7th IEEE Computer Society Foundations Workshop, pages 192-200. IEEE Computer Society, 1994.

[13] John Clark and Jeremy Jacob. A survey of authentication protocol literature: Version 1.0, 1997.

[14] H. Comon-Lundh and V. Cortier. Security properties: two agents are sufficient. In Proceedings of ESOP'2003, LNCS 2618, pages 99-113. Springer, 2003.

[15] Christopher Dilloway and Gavin Lowe. On the specification of secure channels. In Proceedings of WITS'07, 2007.

[16] Danny Dolev and Andrew C. Yao. On the security of public key protocols. IEEE Trans. Inform. Theory, 29(2):198-208, 1983.

[17] Allaa Kamil and Gavin Lowe. Specifying and modelling secure channels in strand spaces. In Proceedings of FAST'09, LNCS 5983, pages 233-247. Springer, 2010.

[18] Jonathan K. Millen and Grit Denker. Capsl and mucapsl. Journal of Telecommunications and Information Technology, 4:16-27, 2002.

[19] Sebastian Mödersheim, Luca Viganò, and David A. Basin. Constraint differentiation: Search-space reduction for the constraint-based analysis of security protocols. Journal of Computer Security, 18(4):575-618, 2010.

[20] Patrick Schaller, Benedikt Schmidt, David Basin, and Srdjan Capkun. Modeling and verifying physical properties of security protocols for wireless networks. In Proceedings of the 22nd IEEE Computer Security Foundations Symposium. IEEE Computer Society, 2009.

[21] Paul Syverson, Catherine Meadows, and Iliano Cervesato. Dolev-Yao is no better than Machiavelli. In First Workshop on Issues in the Theory of Security WITS'00, pages 87-92, 2000.

\section{A Extended tables for BME}

In this appendix, we present a detailed view of the outcome of an attack carried out against $\mathrm{BME}$ and involving only the non-collaborative attackers $E_{1}$ and $E_{2}$. Refer to Section 3 for a definition of BME, attacker behavior against BME, attack traces and cases.

Note that in cases 1, 2 and 3 (shown in Table 6), $E_{j}$ 's request is the $j$-th served by $S$. In cases 4,5 and $6, E_{2}$ is the attacker with knowledge advantage. For clarity, for 
cases 4 and 6 (see Table 7) we mark as $E_{j} *$ the case in which $E_{j}$ 's request is served first by $S$. In case $5, E_{2}$ 's request is the only served and the distinction is unnecessary.

A competitive attacker $E$ attacking BME can:

- succeed and compromise a key that $A$ will use;

- fail and realize it (by timeout);

- fail without realizing it, by acquiring the wrong key;

- fail without realizing it, even though $E$ acquired the right key.

Honest agents under attack can:

- detect the attack and abandon the protocol before carrying out step (3);

- realize that the key has been compromised and keep safe by not using it;

- fail to detect an attack but use their keys safely, because all attackers have failed to acquire the correct key;

- use a compromised key.

Attackers who realize their failure can infer the following:

$\alpha$ Mislabeled or unknown attacker. The attacker spies two messages from $S$ and none from $A$ in response; he deduces that $A$ had opened a single session and that at least one request to $S$ (in addition to his own) was an attack. The attacker realizes that he has either mislabeled as honest one of the active attackers or that an unknown competitor is active.

$\beta$ Unknown attacker. The attacker spies two messages from $S$ and none from $A$ in response; he deduces that $A$ had opened a single session and that at least one request to $S$ (in addition to his own) was an attack. However, he has seen no additional requests of the type $(A, X)$ transit on the network; the attacker realizes that an unknown competitor is active on the network.

$\gamma$ Missed message: mislabeled or unknown attacker. The attacker spies only one message from $S$ but no reply from $A$; all messages from $S$ that successfully reach $A$ are seen, so the attacker deduces that he has missed $A$ 's response. Thus, an active competitor (mislabeled or unknown) has erased it, preventing the attacker from acquiring it through the spy rule.

$\delta$ Missed message. Similar to case $\gamma$. The attacker does not draw further conclusions because he is already aware of an active attacker that may have erased the message.

$\varepsilon$ Suspect condemned. The attacker $E$ has put to test the dishonesty of an agent X in $U_{E}$ (the suspect). Failing the attack is interpreted as a confirmation that the suspect is dishonest: $X$ is placed into $A_{E}$. 


\begin{tabular}{|c|c|c|c|c|c|c|c|c|}
\hline Trace & canSee & Agent & Result & $\begin{array}{l}\text { Belief } \\
\end{array}$ & Key & \multicolumn{2}{|c|}{ Detection } & Guardian \\
\hline $\begin{array}{ll}\text { T1 } \\
\end{array}$ & & & & & & Case 1 & $\begin{array}{l}\text { Case } 3 \\
\end{array}$ & \\
\hline & - & $\begin{array}{l}E_{1} \\
E_{2} \\
A\end{array}$ & $\begin{array}{l}\text { failure } \\
\text { failure } \\
\text { safe }\end{array}$ & $\begin{array}{l}\text { failure } \\
\text { failure } \\
\text { attack }\end{array}$ & $\begin{array}{c}\text { none } \\
\text { none } \\
\text { not used }\end{array}$ & $\begin{array}{c}\alpha \\
\alpha \\
2 \text { keys }\end{array}$ & $\begin{array}{c}\beta \\
\beta \\
2 \text { keys }\end{array}$ & $\begin{array}{l}\text { of help } \\
\text { of help }\end{array}$ \\
\hline T2 & step (3) & & & & & Case 1 & Case 3 & \\
\hline & $\left\{E_{1}, E_{2}\right\}$ & $\begin{array}{c}E_{1} \\
E_{2} \\
A\end{array}$ & $\begin{array}{c}\text { Success } \\
\text { failure } \\
\text { attacked }\end{array}$ & $\begin{array}{c}\text { success } \\
\text { success } \\
\text { safe }\end{array}$ & $\begin{array}{c}\text { right } \\
\text { wrong } \\
\text { broken }\end{array}$ & $\begin{array}{l}\text { none } \\
\text { none } \\
\text { none }\end{array}$ & $\begin{array}{l}\text { none } \\
\text { none } \\
\text { none }\end{array}$ & $\begin{array}{l}\text { of help } \\
\text { no effect }\end{array}$ \\
\hline & $\left\{E_{1}\right\}$ & $\begin{array}{c}E_{1} \\
E_{2} \\
A \\
\end{array}$ & $\begin{array}{c}\text { success } \\
\text { failure } \\
\text { attacked }\end{array}$ & $\begin{array}{l}\text { success } \\
\text { failure } \\
\text { safe }\end{array}$ & $\begin{array}{c}\text { right } \\
\text { none } \\
\text { broken } \\
\end{array}$ & $\begin{array}{c}\text { none } \\
\gamma \\
\text { none } \\
\end{array}$ & $\begin{array}{c}\text { none } \\
\gamma \\
\text { none }\end{array}$ & $\begin{array}{c}\text { of help } \\
\text { no effect }\end{array}$ \\
\hline & $\left\{E_{2}\right\}$ & $\begin{array}{c}E_{1} \\
E_{2} \\
A\end{array}$ & $\begin{array}{c}\text { failure } \\
\text { failure } \\
\text { safe }\end{array}$ & $\begin{array}{c}\text { failure } \\
\text { success } \\
\text { safe }\end{array}$ & $\begin{array}{c}\text { none } \\
\text { wrong } \\
\text { used }\end{array}$ & $\begin{array}{c}\gamma \\
\text { none } \\
\text { none }\end{array}$ & $\begin{array}{c}\gamma \\
\text { none } \\
\text { none }\end{array}$ & $\begin{array}{l}\text { of help } \\
\text { of help }\end{array}$ \\
\hline T3 & step (3) & & & & & Case 1 & Case 3 & \\
\hline & $\left\{E_{1}, E_{2}\right\}$ & $\begin{array}{l}E_{1} \\
E_{2} \\
A \\
\end{array}$ & $\begin{array}{l}\text { failure } \\
\text { failure } \\
\text { safe }\end{array}$ & $\begin{array}{c}\text { success } \\
\text { success } \\
\text { attack }\end{array}$ & $\begin{array}{c}\text { right } \\
\text { wrong } \\
\text { not used }\end{array}$ & $\begin{array}{c}\text { none } \\
\text { none } \\
2 \text { keys } \\
\end{array}$ & $\begin{array}{c}\text { none } \\
\text { none } \\
2 \text { keys }\end{array}$ & $\begin{array}{l}\text { of help } \\
\text { of help }\end{array}$ \\
\hline & $\left\{E_{1}\right\}$ & $\begin{array}{c}E_{1} \\
E_{2} \\
A\end{array}$ & $\begin{array}{l}\text { failure } \\
\text { failure } \\
\text { safe }\end{array}$ & $\begin{array}{l}\text { success } \\
\text { failure } \\
\text { attack }\end{array}$ & $\begin{array}{c}\text { right } \\
\text { none } \\
\text { not used }\end{array}$ & $\begin{array}{c}\text { none } \\
\gamma \\
2 \text { keys }\end{array}$ & $\begin{array}{c}\text { none } \\
\gamma \\
2 \text { keys }\end{array}$ & $\begin{array}{l}\text { of help } \\
\text { of help }\end{array}$ \\
\hline & $\left\{E_{2}\right\}$ & $\begin{array}{c}E_{1} \\
E_{2} \\
A\end{array}$ & $\begin{array}{c}\text { failure } \\
\text { failure } \\
\text { safe }\end{array}$ & $\begin{array}{c}\text { failure } \\
\text { success } \\
\text { attack }\end{array}$ & $\begin{array}{c}\text { none } \\
\text { wrong } \\
\text { not used }\end{array}$ & $\begin{array}{c}\gamma \\
\text { none } \\
2 \text { keys }\end{array}$ & $\begin{array}{c}\gamma \\
\text { none } \\
2 \text { keys }\end{array}$ & $\begin{array}{l}\text { of help } \\
\text { of help }\end{array}$ \\
\hline T4 & - & & & & & & & \\
\hline & & $\begin{array}{l}E_{1} \\
E_{2} \\
A \\
\end{array}$ & $\begin{array}{c}\text { failure } \\
\text { failure } \\
\text { safe } \\
\end{array}$ & $\begin{array}{l}\text { failure } \\
\text { failure } \\
\text { attack } \\
\end{array}$ & $\begin{array}{l}\text { none } \\
\text { none } \\
\text { none }\end{array}$ & $\begin{array}{r}\text { correct } \\
\text { correct } \\
\text { no an } \\
\end{array}$ & $\begin{array}{l}\text { erstanding } \\
\text { erstanding } \\
\text { er: Dos }\end{array}$ & $\begin{array}{l}\text { of help } \\
\text { of help }\end{array}$ \\
\hline T5 & step (3) & & & & & & & \\
\hline & $\left\{E_{1}, E_{2}\right\}$ & $\begin{array}{l}E_{1} \\
E_{2} \\
A\end{array}$ & $\begin{array}{c}\text { failure } \\
\text { success } \\
\text { attacked }\end{array}$ & $\begin{array}{c}\text { success } \\
\text { success } \\
\text { safe }\end{array}$ & $\begin{array}{c}\text { wrong } \\
\text { right } \\
\text { broken }\end{array}$ & correct & $\begin{array}{l}\text { ne } \\
\text { erstanding } \\
\text { ne }\end{array}$ & $\begin{array}{l}\text { no effect } \\
\text { of help }\end{array}$ \\
\hline & $\left\{E_{1}\right\}$ & $\begin{array}{c}E_{1} \\
E_{2} \\
A\end{array}$ & $\begin{array}{c}\text { failure } \\
\text { failure } \\
\text { safe }\end{array}$ & $\begin{array}{l}\text { success } \\
\text { failure } \\
\text { safe }\end{array}$ & $\begin{array}{l}\text { wrong } \\
\text { none } \\
\text { in use }\end{array}$ & & & $\begin{array}{l}\text { of help } \\
\text { of help }\end{array}$ \\
\hline & $\left\{E_{2}\right\}$ & $\begin{array}{l}E_{1} \\
E_{2} \\
A\end{array}$ & $\begin{array}{c}\text { failure } \\
\text { success } \\
\text { attacked }\end{array}$ & $\begin{array}{c}\text { failure } \\
\text { success } \\
\text { safe }\end{array}$ & $\begin{array}{c}\text { none } \\
\text { right } \\
\text { broken }\end{array}$ & correct & erstanding & $\begin{array}{l}\text { no effect } \\
\text { of help }\end{array}$ \\
\hline
\end{tabular}

Table 6: Outcomes of a competitive attack against BME involving the attackers $E_{1}$ and $E_{2}$ and the honest initiator $A$ (cases 1, 2, 3 and 5). Traces are described in Table 4 canSee () describes the set of attackers who spy the message sent by $A$ at step (3); for each role, we report the actual result of the attack (result), if the agent believes he has succeeded or failed (belief) and whether he has acquired the right key, the wrong key or no key at all (key). When attackers realize their failure, they can infer the reason for failing as shown in the column Detection; the honest agent $A$ can detect ongoing attacks by receiving two answers from $S$ or none. In the last column, we show the result of introducing a guardian agent, playing the role in the corresponding row against an attacker playing the other role.

\section{B A case study: the Shamir-Rivest-Adleman Three-Pass Protocol}

The Shamir-Rivest-Adleman Three-Pass protocol (SRA3P), described in [13], has been proposed to transmit data securely on insecure channels, bypassing the difficulties connected to the absence of prior agreements between the agents $A$ and $B$ to establish a shared key. The security property targeted by SRA3P is confidentiality; if the message transmitted is interpreted as a session key, then the protocol can be considered as a key transport protocol.

SRA3P relies on the assumption that the kind of cryptography employed is commutative, i.e. that $\left\{\left|\{|M|\}_{K_{A}}\right|\right\}_{K_{B}}=\left\{\left|\{|M|\}_{K_{B}}\right|\right\}_{K_{A}}$ holds. We use the standard notation for symmetric cryptography to emphasize commutativity. The protocol consists in three message exchanges, as shown in Table $8 \mathrm{~A}$.

The classical attack to SRA3P exploits $A$ as an oracle for the content of the message (Table $8 \mathrm{~B}$ ). The attacker $E$ replaces the intended recipient $B$ in receiving the message and pretends to perform step (2) - in actuality sending back the message $\{|M|\}_{K_{A}}$ with- 


\begin{tabular}{|c|c|c|c|c|c|c|c|c|}
\hline Trace & canSee & Agent & Result & Belief & Key & Dete & ction & Guardian \\
\hline T1 & - & & & & & Case 4 & $\begin{array}{l}\text { Case } 6 \\
\end{array}$ & \\
\hline & & $E_{1}{ }^{*}$ & failure & failure & none & $\beta$ & $\beta$ & of help \\
\hline & & $E_{1}$ & failure & failure & none & $\beta$ & $\beta$ & of help \\
\hline & & $E_{2}{ }^{*}$ & failure & failure & none & $\alpha$ & $\varepsilon$ & of help \\
\hline & & $E_{2}$ & failure & failure & none & $\alpha$ & $\varepsilon$ & of help \\
\hline & & $A$ & & attack & not used & 2 keys & 2 keys & \\
\hline T2 & step (3) & & & & & Case 4 & $\begin{array}{l}\text { Case } 6 \\
\end{array}$ & \\
\hline & $\left\{E_{1}, E_{2}\right\}$ & $E_{1}{ }^{*}$ & success & success & right & none & none & of help \\
\hline & & $E_{1}$ & failure & success & wrong & none & none & no effect \\
\hline & & $E_{2}{ }^{*}$ & success & success & right & none & none & of help \\
\hline & & $E_{2}$ & failure & success & wrong & none & none & no effect \\
\hline & $\left\{E_{1}\right\}$ & $\frac{A}{E_{1} *}$ & $\begin{array}{l}\text { attacked } \\
\text { success }\end{array}$ & $\frac{\text { sate }}{\text { success }}$ & $\frac{\text { broken }}{\text { right }}$ & $\begin{array}{l}\text { none } \\
\text { none }\end{array}$ & $\begin{array}{l}\text { none } \\
\text { none }\end{array}$ & of help \\
\hline & & $E_{1}$ & failure & success & wrong & none & none & of help \\
\hline & & $E_{2} *$ & failure & failure & none & $\gamma$ & $\varepsilon$ & of help \\
\hline & & $E_{2}$ & failure & failure & none & $\gamma$ & $\varepsilon$ & no effect \\
\hline & & $A$ & attacked & safe & broken & none & none & \\
\hline & $\left\{E_{2}\right\}$ & $E_{1}{ }^{*}$ & failure & failure & none & $\gamma$ & $\gamma$ & of help \\
\hline & & $E_{1}$ & failure & failure & none & $\gamma$ & $\gamma$ & no effect \\
\hline & & $E_{2} *$ & success & success & right & none & none & of help \\
\hline & & $E_{2}$ & failure & success & wrong & none & none & \\
\hline & & $A$ & safe & safe & used & none & none & \\
\hline T3 & step (3) & & & & & Case 4 & Case 6 & \\
\hline & $\left\{E_{1}, E_{2}\right\}$ & $E_{1}{ }^{*}$ & failure & success & right & none & none & of help \\
\hline & & $E_{1}$ & failure & success & wrong & none & none & of help \\
\hline & & $E_{2}{ }^{*}$ & failure & success & right & none & none & of help \\
\hline & & $E_{2}$ & failure & success & wrong & none & none & of help \\
\hline & & $A$ & safe & attack & not used & 2 keys & 2 keys & \\
\hline & $\left\{E_{1}\right\}$ & $E_{1}{ }^{*}$ & failure & success & right & none & none & of help \\
\hline & & $E_{1}$ & failure & success & wrong & none & none & of help \\
\hline & & $E_{2}{ }^{*}$ & failure & failure & none & $\gamma$ & $\varepsilon$ & of help \\
\hline & & $E_{2}$ & failure & failure & none & $\gamma$ & $\varepsilon$ & of help \\
\hline & & $A$ & safe & attack & not used & 2 keys & 2 keys & \\
\hline & $\left\{E_{2}\right\}$ & $E_{1}{ }^{*}$ & & failure & & $\gamma$ & $\gamma$ & of help \\
\hline & & $E_{1}$ & failure & failure & none & $\gamma$ & $\gamma$ & of help \\
\hline & & $E_{2}{ }^{*}$ & failure & success & right & none & none & of help \\
\hline & & $E_{2}$ & failure & success & wrong & none & none & of help \\
\hline & & $A$ & safe & attack & not used & 2 keys & 2 keys & \\
\hline
\end{tabular}

Table 7: Outcomes of a competitive attack against BME involving the attackers $E_{1}$ and $E_{2}$ and the honest initiator $A$ (cases 4 and 6). $E_{j}{ }^{*}: E_{j}$ 's request at step $\left(1_{i}\right)$ is served by $S$ first. Traces are described in Table 4, canSee() describes the set of attackers who spy the message sent by $A$ at step (3); for each role, we report the actual result of the attack (result), if the agent believes he has succeeded or failed (belief) and whether he has acquired the right key, the wrong key or no key at all (key). When attackers realize their failure, they can infer the reason for failing as shown in the column Detection; the honest agent $A$ can detect ongoing attacks by receiving two answers from $S$ or none. In the last column, we show the result of introducing a guardian agent playing the role in the corresponding row against an attacker playing the other role.

out further encryption. A continues according to the protocol and removes his key from the message, thus sending back the secret $M$ without any encryption. We represent the message as $M^{*}$ to emphasize that $M$ transits in the clear without $A$ meaning it.

The classical attack is successful; however, it prevents the intended recipient $B$ from receiving any messages at all. In case the honest agents had prior agreement that an exchange was to take place, $B$ can detect that something has gone wrong. The classical attack is very strong against detection even in this case: after discovering $M$, the attacker $E$ impersonates $A$ and performs the protocol with $B$, de facto carrying out a complete man-in-the-middle attack. In this manner, the attack on SRA3P goes completely undetected and the attacker gains access to the secret key $M$.

Provided that some attacker answered $A$ in step (2) by sending $\{|M|\}_{K_{A}}$, it is sufficient to spy the message in step (3) to acquire the secret. In our set-up, any attacker attempting to erase a message is always successful in preventing honest agents from receiving it, but he is not necessarily successful in hiding it from other attackers (all attackers in canSee $\left(\left\langle A, M^{*}, B\right\rangle, i\right)$ have access to $\left.M\right)$. In this situation, $E_{2}$ can prevent his competitors from acquiring the secret only by weakening their ability to identify the 
message $M^{*}$ as the true response of $A$ in step (3). A competitive attacker will therefore attempt to mislead his competitors by sending on the network fake messages that are in no way related to the information coming from the initiator $A$.

\begin{tabular}{|c|c|c|c|c|c|}
\hline \multicolumn{3}{|c|}{ (A) SRA3P } & \multicolumn{3}{|c|}{ (B) Classical Attack } \\
\hline $\begin{array}{l}(1) \\
(2) \\
(3)\end{array}$ & \multicolumn{2}{|c|}{$\begin{aligned} A \rightarrow B & :\{|M|\}_{K_{A}} \\
B \rightarrow A & :\left\{\left|\{|M|\}_{K_{A}}\right|\right\}_{K_{B}} \\
A \rightarrow B & :\{|M|\}_{K_{B}}\end{aligned}$} & \multicolumn{3}{|c|}{$\begin{array}{lll}\text { (1) } & A \rightarrow E(B) & :\{|M|\}_{K_{A}} \\
\text { (2) } & E(B) \rightarrow A & :\{|M|\}_{K_{A}} \\
\text { (3) } & A \rightarrow E(B) & : M^{*}=M\end{array}$} \\
\hline \multicolumn{3}{|c|}{ (C) Strong attack } & \multicolumn{3}{|c|}{ (D) Competitive attack } \\
\hline $\begin{array}{l}(1) \\
(2) \\
\left(3^{\prime}\right) \\
(3)\end{array}$ & $\begin{array}{l}A \rightarrow E_{1,2}(B) \\
E_{1}(B) \rightarrow A \\
E_{2}(A) \rightarrow E_{1} \\
A \rightarrow E_{1,2}(B)\end{array}$ & $\begin{array}{l}:\{|M|\}_{K_{A}} \\
:\{|M|\}_{K_{A}} \\
: M_{\text {fake }} \\
: M^{*}\end{array}$ & $\begin{array}{l}(1) \\
(2) \\
\left(3^{\prime}\right) \\
(3)\end{array}$ & $\begin{array}{l}A \rightarrow E_{1,2}(B) \\
E_{1}(B) \rightarrow A \\
E_{2}(A) \rightarrow E_{1}(B) \\
A \rightarrow E_{1,2}(B)\end{array}$ & $\begin{array}{l}:\{|M|\}_{K_{A}} \\
:\{|M|\}_{K_{A}} \\
: M_{\text {fake }} \\
: M^{*}\end{array}$ \\
\hline
\end{tabular}

Table 8: Attacks against the Shamir-Rivest-Adelman Three-Pass Protocol (SRA3P). $K_{A}$ and $K_{B}$ are private keys and cryptography is commutative. (A): Protocol followed by honest agents. (B): Classical attack on SRA3P, employed by attackers when unaware of active competitors. (C): Strong non-collaborative attack, employed by attackers when the competitor's identifier is known ( $E_{2}$ knows that his competitor is $\left.E_{1}\right)$. (D): Competitive attack, employed by attackers when aware of the existence of an active competitor but unsure of the competitor's identity $\left(E_{2}\right.$ knows that he has a competitor but does not know that it is $E_{1}$ ).

If the recipient of a fake message is expecting to receive $M^{*}$, he may be led into thinking that he has successfully carried out his attack. He may then stop spying the current run of the conversation between $A$ and $B$ and conclude that he has succeeded when in fact he has acquired the wrong "secret" $M_{\text {fake }}$. If, instead, the competitor $E_{1}$ is not following the classical attack and chooses to keep listening in on the conversation, he receives more than one message playing the role of $M^{*}$ and does not know which one has been sent by the honest agent $A$.

The competitor faces a degree of uncertainty in identifying $M^{*}$ that is not present in the classical attack. The degree of uncertainty to which $E_{1}$ is subject can be increased arbitrarily by $E_{2}$, who can send multiple and unrelated fake messages, both before and after $M^{*}$ transits on the network. This style of attack grows in effectiveness as $E_{2}$ is better able to construct misleading fake messages.

The success of this non-collaborative behavior in securing sole ownership of the secret depends critically on the listening behavior of the competitor: if $E_{1}$ stops spying network traffic as soon as a response is received, then it is critical for $E_{2}$ to send a fake message before A's reply; in case of success, the competitor fails to acquire the secret. If the competitor is actively listening past the reception of the first response, then $M^{*}$ is eventually acquired - but not by itself: a situation of uncertainty arises.

In classical settings, uncertainty does little more than affect the probability that an attack will be successful; however, if honest agents are immersed in a retaliatory framework, guessing the wrong $M^{*}$ and using it as a session key to communicate with $A$ could have significant consequences. Therefore, attackers in non-collaborative scenarios should be careful to evaluate the probability of correctly guessing $M^{*}$ against the added costs of failure - either in terms of retaliation or of the strategic risks of being detected or identified by honest agents.

As a result of this discussion, for competitive scenarios involving SRA3P, we propose two variants of the classical attack, employed by attackers who are aware of the 
presence of active competitors. We term the variants strong and competitive attack, differing with respect to attacker knowledge. If the attacker is aware of the identity of the competitor, he will employ the strong attack, whereas he will resort to the competitive attack when only the competitor's presence is known. These new attack behaviors are also oracle-type (transmission step, see [12] for a taxonomy of flaws and attacks) and are shown in Table $8 \mathrm{C}$ and $8 \mathrm{D}$.

The main difference between the two non-collaborative attack behaviors lies in the method of delivery of fake messages to $E_{1}$. If the competitor's identity is known, $E_{2}$ can ensure that the fake message is seen even if $E_{1}$ is not paying attention to $E_{2}$ 's traffic: $E_{2}$ sends the fake message directly, using the network primitive send. If, on the other hand, $E_{1}$ 's identity is unknown, $E_{2}$ is forced to rely on a reasonable prediction of $E_{1}$ 's behavior and thus injects the fake message, impersonating $A$. The misleading message $M_{\text {fake }}$ is successfully delivered if $E_{2}$ is present in $E_{1}$ 's dataset and $E_{1}$ spies it. If $E_{1}$ does not gain $M_{\text {fake }}, E_{2}$ fails to pollute the competitor's knowledge but does not compromise his own ability to observe $M^{*}$

SRA3P is such that all attentive attackers can potentially acquire the secret if an attack on the initiator $A$ is carried out. Exclusive knowledge of the secret can only occur through two mechanisms: through the outcome of erase requests (which is not under the control of network agents) or by misleading other attackers into interpreting a fake message as $M^{*}$.

An attack is successful if it goes undetected by the initiator $A$, who then transmits $M$ in the clear as $M^{*}$. Our agents are intelligent and they make use of all information available to perform in-protocol detection of attacks. With respect to SRA3P, a clear indication for $A$ consists in receiving a duplicate response from agents posing as $B$; under this circumstance, $A$ concludes that there has been a security violation and halts the execution of the protocol to protect the secret $M$.

From the attackers' perspective, an ongoing attack can be detected by observing that the message transiting on the network in step (2) is equal to the message $\{|M|\}_{K_{A}}$ transiting on step (1). The attack trace is unambiguous to spying attackers. SRA3P is very unfriendly for attacker labeling: identifiers do not transit on the network, neither in the clear nor encrypted. Decisional processes cannot rely on any conclusive information concerning the identity of the agents involved in a given protocol run and must resort to inference on the basis of their current knowledge.

\section{B.1 Attacker configuration and outcomes of interaction}

We examine the outcome of attacks carried out in a non-collaborative environment in six cases, corresponding to different conditions of knowledge and belief for two attackers, $E_{1}$ and $E_{2}$. Refer to Table 9 for a synthetic view of the message exchanges in each configuration. In order to completely specify agent behavior, we state the following:

1. An attacker who spies, before starting his own attack, the attack trace $\{|M|\}_{K_{A}}$ transiting on the network moves on to step (3) of his chosen attack (strong or competitive). If the attacker spies the attack trace after sending $\{|M|\}_{K_{A}}$ himself, then he requests that the message be erased. In our set-up, an erase-request always prevents the message from reaching its honest recipient, although other attackers cannot deterministically be prevented from spying it. This behavioral rule accounts for attackers being aware that duplicate messages can be exploited to perform attack detection. 
2. An attacker that is employing a competitive attack (as he is aware of the presence of active competitors) continues spying on the network even after receiving the first message.

3. An attacker may learn that he has incorrectly classified an agent as honest. We do not wish to focus here on decisional processes for agent classification and therefore we posit that the decisional process is an oracle for the identifier of the mislabeled agent. We stipulate that, whenever evidence that an agent has been mislabeled arises, the decisional processes of the agents allow relabeling in $A$ the dishonest agent who has triggered the anomalous situation detected. For completeness, we explicitly mention in case 1.T1-B which choices would be available to the agent, should the decisional process yield incorrect answers.

4. We posit that $\operatorname{canSee}()$ for $A$ 's opening message comprises both $E_{1}$ and $E_{2}$; if this were not the case, only one attacker would be active in the run of the protocol examined.

5. We postulate that $\operatorname{canSee}()$ yields the entire attacker set for the message sent in step (3) by the honest agent $A$. If this were not the case, then only some (one) of the intruders could acquire $M^{*}$. For the sake of concisiveness, in the rest of this section we discuss explicitly only the situation posited. Refer to Section C for detailed analysis of how outcomes are affected by $\operatorname{canSee}($ ).

Case 1: $E_{1}$ and $E_{2}$ know each other as honest.

$E_{1}$ and $E_{2}$ know each other's identifiers (i.e. they are paying attention to each other: $E_{1} \in D_{E_{2}}$ and $E_{2} \in D_{E_{1}}$ ), but they are both mistaken in that they have labeled the other as honest $\left(E_{1} \in H_{E_{2}}\right.$ and $E_{2} \in H_{E_{1}}$ ). Initially, $E_{1}$ and $E_{2}$ are unaware of active competitors and mount the classical attack The first between $E_{1}$ and $E_{2}$ to send the message at step (2) reveals to the other that he has incorrectly classified an agent. Without loss of generality, let us suppose that $E_{1}$ attacks first. $E_{2}$ employs his decisional processes to identify the mislabeled attacker.

(1.T1-A): $E_{1}$ is identified as an attacker by $E_{2} . E_{2}$ switches to the strong attack, with the goal of gaining exclusive access to $M$. In step $\left(3_{2}\right), E_{2}$ sends a fake message to the unsuspecting competitor $E_{1}$, who is expecting a message from $A$ containing $M^{*}$ on the clear. $E_{1}$ may now think that he has successfully completed the attack, but in fact he did not acquire the secret $M$. After receiving $M_{\text {fake }}, E_{1}$ stops monitoring the network, according to the classical attack behavior.

If $E_{1}$ continues to spy, he will also acquire $M^{*}$. However, $E_{1}$ finds himself in a situation of uncertainty, as he is not able to determine if it is $M_{\text {fake }}$ or $M^{*}$ (or neither) that comes from $A . E_{1}$ can at most determine that there is an unlabeled active competitor, one that he has not previously identified in $A_{E_{1}}$.

(1.T1-B): $E_{2}$ fails to identify $E_{1}$ as a dishonest agent. $E_{2}$ has two strategies available: i) risk revealing himself as an attacker and employ the strong attack against all agents he is attentive to (with the exception of the initiator of the protocol); ii) employ the competitive attack with partial impersonification.

\section{Case 2: $E_{1}$ and $E_{2}$ know each other as attackers.}

Both $E_{1}$ and $E_{2}$ (correctly) think that there are active competitors; they know the competitor's identity and thus both follow the strong attack. The attack trace prescribes waiting for a competitor to start the attack procedure, by sending $\{|M|\}_{K_{A}}$ to $A$. Both 
attackers are waiting for the other to take action. The situation could result in a deadlock, but the attackers know that a message has been erased and that $A$ is waiting for an answer.

The attackers wait for a reasonable amount of time and then one takes the initiative. Let us suppose that it is $E_{2}$ who first answers $A$. The strong attack consists in polluting the knowledge base of the competitor with a fake message. Both attackers send their fake messages $\left(M^{\prime}\right.$ and $\left.M^{\prime \prime}\right)$, thereby recreating the uncertainty of the previous case. This time the uncertainty spreads over both attackers and none dominates the other.

\begin{tabular}{|c|c|c|c|c|c|}
\hline \multicolumn{3}{|c|}{ T1: cases $1,4,5,6$} & \multicolumn{3}{|c|}{ T2: case 2} \\
\hline $\begin{array}{l}(1) \\
\left(2_{1}\right) \\
\left(3_{2}\right) \\
(3)\end{array}$ & $\begin{array}{l}A \rightarrow E_{1,2}(B) \\
E_{1}(B) \rightarrow A \\
E_{2}(A) \rightarrow E_{1} \\
A \rightarrow E_{[1], 2}(B)\end{array}$ & $\begin{array}{l}:\{|M|\}_{k_{A}} \\
:\{|M|\}_{k_{A}} \\
: M_{\text {fake }} \\
: M^{*}\end{array}$ & $\begin{array}{l}(1) \\
(2) \\
\left(3_{1}\right) \\
\left(3_{2}\right) \\
(3)\end{array}$ & $\begin{array}{l}A \rightarrow E_{1,2}(B) \\
E_{2}(B) \rightarrow A \\
E_{1}(A) \rightarrow E_{2} \\
E_{2}(A) \rightarrow E_{1} \\
A \rightarrow E_{1,2}(B)\end{array}$ & $\begin{array}{l}:\{|M|\}_{k_{A}} \\
:\{|M|\}_{k_{A}} \\
: M^{\prime} \\
: M^{\prime \prime} \\
: M^{*}\end{array}$ \\
\hline \multicolumn{3}{|c|}{ T3: case 3} & \multicolumn{3}{|c|}{ T4: cases 4,5 and 6} \\
\hline $\begin{array}{l}(1) \\
\left(2_{1}\right) \\
\left(2_{2}\right)\end{array}$ & $\begin{array}{l}A \rightarrow E_{1,2}(B) \\
E_{1}(B) \rightarrow A \\
E_{2}(B) \rightarrow A\end{array}$ & $\begin{array}{l}:\{|M|\}_{k_{A}} \\
:\{|M|\}_{k_{A}} \\
:\{|M|\}_{k_{A}}\end{array}$ & $\begin{array}{l}(1) \\
\left(2_{2}\right) \\
\left(2_{1}\right) \\
\left(3_{2}\right) \\
(3)\end{array}$ & $\begin{array}{l}A \rightarrow E_{1,2}(B) \\
E_{2}(B) \rightarrow A \\
E_{1}(B) \rightarrow E_{2}(A) \\
E_{2}(A) \rightarrow E_{1} \\
A \rightarrow E_{1,[2]}(B)\end{array}$ & $\begin{array}{l}:\{|M|\}_{k_{A}} \\
:\{|M|\}_{k_{A}} \\
:\{|M|\}_{k_{A}} \\
: M_{\text {fake }} \\
: M^{*}\end{array}$ \\
\hline
\end{tabular}

Table 9: Traces for non-collaborative attacks against SRA3P. Traces are exhaustive aside for order of attackers. Case 1: $E_{1}$ and $E_{2}$ know each other as honest. Case 2: $E_{1}$ and $E_{2}$ know each other as dishonest. Case 3: $E_{1}$ and $E_{2}$ are unaware of each other. Case 4: $E_{2}$ knows $E_{1}$ as honest. Case 5: $E_{2}$ knows $E_{1}$ as dishonest. Case 6: $E_{2}$ knows $E_{1}$ but has not yet established a belief on $E_{1}$ 's honesty.

Case 3: $E_{1}$ and $E_{2}$ are unaware of each other.

$E_{1}$ and $E_{2}$ are unaware of the other's presence - i.e. they are not paying attention to the other's activity $\left(E_{1} \notin D_{E_{2}}\right.$ and $\left.E_{2} \notin D_{E_{1}}\right)$. Thus, both $E_{1}$ and $E_{2}$ employ the classical attack. The attackers, not paying attention to the other's communications, do not realize that an attack trace is transiting on the network. A receives a duplicate message, that he correctly interprets in terms of an ongoing attack. The attackers are detected, even if not explicitly identified. $A$ abandons the protocol to keep the secret $M$ safe.

\section{Case 4: $E_{2}$ knows $E_{1}$ as honest.}

$E_{2}$ is not aware of other attackers and can choose to attack right away or wait a reasonable time to try detecting a mislabeled attacker.

(4.T1): $E_{2}$ waits and $E_{1}$ starts the classical attack. $E_{2}$ has the chance of detecting $E_{1}$ as an attacker and starts the strong attack. The situation is reduced to case 1 . If $E_{1}$ continues to listen on the network after the end of his (unsuccessful) attack, he realizes that he is in a situation of uncertainty, not knowing which between $M^{*}$ and $M_{\text {fake }}$ is $A$ 's secret. $E_{1}$ is now certain that an attacker is present but he doesn't know who, because the identifier $E_{2}$ is not in $E_{1}$ 's proprietary dataset. $E_{1}$ can thus switch to an exploratory strategy, using the inflow-spy rule for the subsequent runs of the protocol.

(4.T4): $E_{2}$ starts the classical attack. Not having $E_{2}$ 's identifier in his dataset, $E_{1}$ does not pay attention to the message and does not notice the attack trace transiting. $E_{1}$ continues his attack and sends $\{|M|\}_{k_{A}}$. In step $\left(2_{1}\right), E_{2}$ detects the dishonesty of $E_{1}$ and switches to the strong attack. There is an important difference with respect to case 
4.T1: $E_{2}$ erases the message sent by $E_{1}$ to $A$, thereby preventing $A$ from detecting a duplicate message and protecting his own attack.

Case 5: $E_{2}$ knows $E_{1}$ as dishonest.

Only one out of the two attackers $E_{1}$ and $E_{2}$ is paying attention to the other and knows his identifier. Here we consider $E_{1} \in A_{E_{2}}$ and $E_{2} \notin D_{E_{1}} . E_{2}$ is sure of the presence of a competitor and knows his identifier. When $A$ initiates the protocol, $E_{2}$ waits for $E_{1}$ to start the attack and prepares to send a fake message in step $\left(3_{2}\right)$, employing the strong attack (case 5.T1). If $E_{1}$ does not send $\{|M|\}_{k_{A}}$ within a reasonable time (case 5.T4), $E_{2}$ performs the attack in step $\left(2_{2}\right)$. This message goes undetected by $E_{1}$, who will send his message at a later point. $E_{2}$ is aware that another attacker is present and is on the watch for a replicate attack message, which he erases. If $E_{1}$ acts first, the sequence of messages is the same as in case 1; otherwise, the sequence is the same as in case 4.T4.

If $E_{1}$ continues to spy after receiving $M_{\text {fake }}$, he can realize that he is in uncertainty with respect to $M$ and can therefore deduce the presence of an unknown attacker. $E_{1}$ moves on to employing exploratory versions of the spy-rules to try gaining information about the identity of the competitor.

Case 6: $E_{2}$ knows $E_{1}$ but he is unsure of $E_{1}$ 's honesty.

Only one out of the two attackers $E_{1}$ and $E_{2}$ is paying attention to the other and knows his identifier. Here we consider $E_{1} \in U_{E_{2}}$ and $E_{2} \notin D_{E_{1}}$. This case reduces to cases 5.T1 and 5.T4, according to who first initiates the attack by sending $\{|M|\}_{k_{A}}$. In case 6.T1, $E_{1}$ opens the attack, whereas in case $6 . T 4$ it is $E_{2}$ who opens. In all cases, $E_{2}$ has a clear advantage because he is paying attention to $E_{1}$ 's messages but his own messages are not being attended to. In addition to what happens in case $5, E_{2}$ has the opportunity to correctly label $E_{1}: E_{2}$ moves $E_{1}$ 's identifier from $U_{E_{2}}$ into $A_{E_{2}}$.

\section{B.2 Success criteria for competitive attackers and honest agents}

Attackers in the SRA3P scenario have a complex success criterion. The best possible result for an attacker consists in i) violating security without the honest agent realizing it and ii) making it such that the other attackers conclude their attacks with false information $\left(M_{\text {fake }}\right.$ taken for $\left.M^{*}\right)$ and without realizing that the information is false. This set of conditions describes an attacker with complete dominance over the system - both over honest agents and over his competitors. As shown for SRA3P (and for BME in Section 3), in competitive scenarios with equal-opportunity attackers it is not possible, in general, to ensure a complete victory under all circumstances.

The result of an attack depends on the strategy and on the knowledge conditions of all the active agents. As a consequence, a competitive agent will try to secure the best result (compatibly with his knowledge of the system) and he will strategically evaluate if it is preferable, for example, to risk being identified as an attacker by other agents or to increase the degree of uncertainty of the competitors. A competitive agent attacking SRA3P evaluates the following factors as part of his success criterion:

1. Success in gaining the secret protected by the security system (or, more generally, in invalidating the target properties of the protocol). Because SRA3P is vulnerable to the classical attack, a single attacker without competition is always successful. The first priority of our competitive attackers is preserving the success of their own attacks, even in the presence of active competitors.

2. Absence of uncertainty on the secret. 
3. Exclusivity in access to the secret.

4. Effects on competitors: denying competitors either access to the secret or certainty on it. The ideal case for a competitive attacker is negating access to the secret and at the same time inducing competitors to think that they have succeeded.

5. Possibility of being identified as an attacker by other attackers. Attackers are aware that knowing the dishonesty of an agent is an advantage, therefore they seek to limit the situations in which they can be detected or identified through an explorative spy-rule. A good example of this strategy is the difference between the two non-collaborative attacks against SRA3P - employing a direct send to the competitor or relying on the prediction that the competitor will spy.

6. Possibility of being identified as an attacker by honest agents.

7. Possibility of identifying competitors - and thus of acquiring a strategic advantage for later runs of the protocol.

An honest agent that uses SRA3P distinguishes five relevant conditions, each associated to a different level of alarm:

1. No attacker has gained the secret and the secret has correctly reached the intended recipient (security). Since SRA3P is vulnerable to attacks, in the presence of attackers this condition never occurs.

2. No attacker has succeeded in gaining the security secret, but the secret has not reached its intended recipient (stalemate, deadlock). For SRA3P, this condition occurs whenever the initiator detects duplicate messages before step (3), e.g. in case 3.

3. One or more attackers have gained the security secret but the honest agent has detected the attack (restart).

4. One or more attackers have gained the security secret, the honest agent has detected the attack and has also acquired new knowledge on the identity of the attacker (retaliate and restart).

5. One or more attackers have gained the security secret but the attack has not been detected (security failure).

During a protocol run, the proprietary datasets evolve in different ways according to the roles and the knowledge of the agents. The interpretation of messages - and along with it the behavior - can vary, both according to prior knowledge on the system and according to strategic considerations.

In Table 10, we show the effects of introducing a guardian $G$ for SRA3P, configured as one of the competitive attackers described in the case study. Compared to the guardian for BME (see Section 4), a guardian for SRA3P appears to be less effective, in that it prevents $E$ from successfully carrying out his attack in fewer cases. However, it must be noted that SRA3P is a much harder protocol to defend because it does not entail that attacker success is mutually exclusive. Remarkably, $G$ can be effective even when he is not aware of $E$ 's presence. The effectiveness of a guardian for SRA3P is comparable to the case of BME, if honest agents can detect and mount retaliatory attacks whenever attackers guess the wrong secret and use it to communicate with honest agents. 


\begin{tabular}{c|cccc}
\hline canSee & Case 2 & Case 3 & $\begin{array}{c}\text { Cases 1+4,5,6 } \\
E \in \text { Attend }_{G}\end{array}$ & $\begin{array}{c}\text { Cases 4,5,6 } \\
\text { G } \in \text { Attend }_{E}\end{array}$ \\
\hline$\{E, G\}$ & $\sim^{+}$ & $\sqrt{ }$ & $\sim$ & \\
$\{G\}$ & $\sqrt{ }$ & $\sqrt{ }$ & $\sqrt{ }$ & $\sqrt{ }$ \\
$\{E\}$ & $\sim^{+}$ & $\sqrt{ }$ & $\sim$ & \\
\hline
\end{tabular}

Table 10: Effects of introducing a guardian $G$ for SRA3P when attacker $E$ is active. $G$ operates according to the same strategy as the attackers in the case study. $G$ 's active interference results in $E$ failing to acquire the secret $(\sqrt{ })$, in being sometimes uncertain $(\sim)$ or in being always uncertain $\left(\sim^{+}\right)$.

\section{Extended tables for SRA3P}

In this appendix, we present a detailed view of the outcome of an attack carried out against SRA3P and involving only the non-collaborative attackers $E_{1}$ and $E_{2}$. Refer to Table 8 for a definition of SRA3P and attacker behavior against SRA3P and to Table 9 for attack traces and cases.

For each case, we report the following subcases (columns):

- attacker $E_{1}$ is using the classical attack and stops spying on the network after receiving the first message that he can interpret as $M$.

- attacker $E_{1}$ continues to spy on the network even after receiving the first message that can be interpreted as $M$, with all possible values of the set canSee() for $A$ 's response in step (3). If an attacker is not in canSee, he fails regardless of the number of fake messages dispatched.

For each attacker role, we describe:

- (Attack) which attack has been used (classical or strong) or if there has been a switch from the classical to the strong attack during the protocol run $(\mathrm{Cl} \rightarrow \mathrm{Str})$.

- (Detection) the ability to acquire further information on competitors. Possible values are: none performed (none); none possible, because the agent already has a correct understanding of the situation (none (c)); in-protocol detection, by spying the attack trace when no competitor is known ((in) trace); post-protocol detection, by realizing that more than one candidate $M$ has been spied and an unknown competitor is responsible for the uncertainty ((post) uncertainty) - with the variant (post $\exists$ ) uncertainty to also signal that the identifier of the previously unknown competitor is not in Attend.

- (Messages) the set of messages that can be interpreted as $M . M$ ! indicates that only $M$ has been spied; $M^{+}$indicates that more than one message, including $M$, has been spied; $M_{\text {fake }}$ that only fake messages have been spied; none, to indicate that no message has been spied during the protocol run.

- (Result) the result of the protocol run. Possible results are: full failure (the attacker does not acquire $M$ and takes a fake message for the secret), failure (the attacker does not acquire $M$ and realizes it), uncertainty (the attacker acquires the secret $M$ along with other fake messages), success (the attacker knows $M$ without uncertainty), dominance (the attacker succeeds and all his competitors fully fail). 
For honest agents we show only the result: either security failure or attack detection through duplicate messages.

The last two rows in each table show the outcomes when a guardian $G$ is introduced along with a single (competitive) attacker $E$. $G_{E_{1}}$ plays the role of $E_{1}$ against $E$ playing $E_{2}$ and $G_{E_{2}}$ plays the role of $E_{2}$ against $E_{1}$. Similarly to attackers, we show for $G$ the possible conclusions that can be drawn on attacker identity and the actual security. Security can be: compromised, if $E$ known $M$ with certainty; uncertain $E$, if $E$ known $M$ but cannot identify it with certainty; restored, if $E$ fails to acquire $M$; enforced, if thanks to $G$ being present, flags were raised for $A$ that allow $A$ to detect an ongoing attack and abort the protocol to protect $M$.

\begin{tabular}{|cc|c|c|c|c|}
\hline \begin{tabular}{cc|c|c|} 
Case 1 \\
Agent
\end{tabular} & Feature & $E_{1}$ stops & \multicolumn{3}{|c|}{$E_{1}$ continues and canSee $\left(M^{*}\right)=$} \\
& & $\left\{E_{1}, E_{2}\right\}$ & $\left\{E_{1}\right\}$ & $\left\{E_{2}\right\}$ \\
\hline \hline$E_{1}$ & Attack & Classical & Classical & Classical & Classical \\
& Detection & none & (post) uncertainty & (post) uncertainty & none \\
& Messages & $M_{\text {fake }}$ & $M^{+}$ & $M^{+}$ & $M_{\text {fake }}$ \\
& Result & full failure & uncertainty & uncertainty & full failure \\
\hline$E_{2}$ & Attack & $\mathrm{Cl} \rightarrow$ Str & $\mathrm{Cl} \rightarrow$ Str & $\mathrm{Cl} \rightarrow$ Str & $\mathrm{Cl} \rightarrow$ Str \\
& Detection & (in) trace & (in) trace & (in) trace & (in) trace \\
& Messages & $M !$ & $M !$ & none & $M !$ \\
& Result & dominance & success & failure & dominance \\
\hline$A$ & Result & failure & failure & failure & failure \\
\hline$G_{E_{1}}$ & Detection & none & (post) label & (post) label & none \\
& Security & compromised & compromised & restored & compromised \\
\hline$G_{E_{2}}$ & Detection & (in) label & (in) label & (in) label & (in) label \\
& Security & restored & uncertain $E$ & uncertain $E$ & restored \\
\hline \hline
\end{tabular}

Table 11: Overall SRA3P results, detailed view of case 1: $E_{1}$ and $E_{2}$ know each other as honest. If $E_{1}$ is no longer listening on the network, only $E_{2}$ can place an erase request in step (3) and thus can acquire the message $M^{*}$ with certainty. If the competitor $E_{1}$ continues to eavesdrop, the dominant intruder can fail to acquire $M^{*}$ whenever $E_{2} \notin$ canSee. If, on the other hand, it is the attacker at disadvantage $\left(E_{1}\right)$ that is not in canSee, then $E_{1}$ fails regardless of the number of fake messages.

\begin{tabular}{|cc|c|c|c|c|}
\hline Case 2 & & $E_{1}$ stops & \multicolumn{3}{|c|}{ canSee $\left(M^{*}\right)=$} \\
Agent & Feature & & $\left\{E_{1}, E_{2}\right\}$ & $\left\{E_{1}\right\}$ & $\left\{E_{2}\right\}$ \\
\hline \hline$E_{1}$ & Attack & - & Strong & Strong & Strong \\
& Detection & - & none (c) & none (c) & none (c) \\
& Messages & - & $M^{+}$ & $M^{+}$ & $M_{\text {fake }}$ \\
& Result & - & uncertainty & uncertainty & full failure \\
\hline$E_{2}$ & Attack & - & Strong & Strong & Strong \\
& Detection & - & none (c) & none (c) & none (c) \\
& Messages & - & $M^{+}$ & $M_{\text {fake }}$ & $M^{+}$ \\
& Result & - & uncertainty & full failure & uncertainty \\
\hline$A$ & Result & - & failure & failure & failure \\
\hline$G_{E_{1}}$ & Detection & - & none (c) & none (c) & none (c) \\
& Security & - & uncertain $E$ & restored & uncertain $E$ \\
\hline$G_{E_{2}}$ & Detection & - & none (c) & none (c) & none (c) \\
& Security & - & uncertain $E$ & uncertain $E$ & restored \\
\hline \hline \multicolumn{7}{|c}{}
\end{tabular}

Table 12: Overall SRA3P results, detailed view of case 2: $E_{1}$ and $E_{2}$ know each other as dishonest. 


\begin{tabular}{|cc|c|}
\hline $\begin{array}{c}\text { Case 3 } \\
\text { Agent }\end{array}$ & Feature & - \\
\hline \hline$E_{1}$ & Attack & Classical \\
& $\begin{array}{c}\text { Detection } \\
\text { Messages } \\
\end{array}$ & $\begin{array}{c}\text { Rest, } \exists \text { ) failure } \\
\text { none } \\
\text { failure }\end{array}$ \\
\hline$E_{2}$ & Attack & Classical \\
& Detection & (post, $\exists$ ) failure \\
& Messages & none \\
& Result & failure \\
\hline$A$ & Result & detection (duplicates) \\
\hline$G_{E_{1}}$ & $\begin{array}{c}\text { Detection } \\
\text { Security }\end{array}$ & (post) $\exists$ \\
& enforced \\
\hline$G_{E_{2}}$ & $\begin{array}{c}\text { Detection } \\
\text { Security }\end{array}$ & (post) $\exists$ \\
& enforced \\
\hline \hline
\end{tabular}

Table 13: Overall SRA3P results, detailed view of case 3: $E_{1}$ and $E_{2}$ are unaware of each other.

\begin{tabular}{|c|c|c|c|c|c|}
\hline \multicolumn{6}{|c|}{$\begin{array}{l}\text { Case 4A: } E_{1} \text { starts the attack } \\
\text { Case 4B: } E_{2} \text { starts the attack }\end{array}$} \\
\hline \multirow[b]{2}{*}{ Agent } & \multirow[b]{2}{*}{ Feature } & \multirow[t]{2}{*}{$E_{1}$ stops } & \multirow{2}{*}{\multicolumn{2}{|c|}{\begin{tabular}{|cc} 
& $\operatorname{canSee}\left(M^{*}\right)=$ \\
$\left\{E_{1}, E_{2}\right\}$ & $\left\{E_{1}\right\}$
\end{tabular}}} & \multirow[b]{2}{*}{$E_{2}$} \\
\hline & & & & & \\
\hline$\overline{E E_{1}}$ & $\begin{array}{c}\text { Attack } \\
\text { Detection } \\
\text { Messages } \\
\text { Result }\end{array}$ & $\begin{array}{c}\text { Classical } \\
\text { none } \\
M_{\text {fake }} \\
\text { full failure }\end{array}$ & $\begin{array}{c}\text { Classical } \\
\text { (post } \exists \text { ) uncertainty } \\
M^{+} \\
\text {uncertainty }\end{array}$ & $\begin{array}{c}\text { Classical } \\
\text { (post } \exists \text { ) uncertainty } \\
M^{+} \\
\text {uncertainty }\end{array}$ & $\begin{array}{c}\text { Classical } \\
\text { none } \\
M_{\text {fake }} \\
\text { full failure }\end{array}$ \\
\hline$E_{2}$ & $\begin{array}{c}\text { Attack } \\
\text { Detection } \\
\text { Messages } \\
\text { Result }\end{array}$ & $\begin{array}{c}\mathrm{Cl} \rightarrow \text { Str } \\
\text { (in) trace } \\
M ! \\
\text { dominance }\end{array}$ & $\begin{array}{c}\mathrm{Cl} \rightarrow \mathrm{Str} \\
\text { (in) trace } \\
M ! \\
\text { success }\end{array}$ & $\begin{array}{c}\mathrm{Cl} \rightarrow \mathrm{Str} \\
\text { (in) trace } \\
\text { none } \\
\text { failure }\end{array}$ & $\begin{array}{c}\mathrm{Cl} \rightarrow \mathrm{Str} \\
\text { (in) trace } \\
M ! \\
\text { dominance }\end{array}$ \\
\hline$A$ & Result & failure & failure & failure & failure \\
\hline$G_{E_{1}}$ & $\begin{array}{l}\text { Detection } \\
\text { Security }\end{array}$ & $\begin{array}{c}\text { none } \\
\text { compromised }\end{array}$ & $\begin{array}{c}\text { post }(\exists) \\
\text { compromised }\end{array}$ & $\begin{array}{l}\text { post }(\exists) \\
\text { restored }\end{array}$ & $\begin{array}{c}\text { none } \\
\text { compromised }\end{array}$ \\
\hline$G_{E_{2}}$ & $\begin{array}{c}\text { Detection } \\
\text { Security }\end{array}$ & $\begin{array}{l}\text { (in) label } \\
\text { restored }\end{array}$ & $\begin{array}{l}\text { (in) label } \\
\text { uncertain } E\end{array}$ & $\begin{array}{l}\text { (in) label } \\
\text { uncertain } E\end{array}$ & $\begin{array}{l}\text { (in) label } \\
\text { restored }\end{array}$ \\
\hline
\end{tabular}

Table 14: Overall SRA3P results, detailed view of case 4: $E_{2}$ knows $E_{1}$ as honest.

\begin{tabular}{|c|c|c|c|c|c|}
\hline \multirow{2}{*}{\multicolumn{6}{|c|}{ Case 5A: $E_{1}$ starts the attack }} \\
\hline & & Case & & & \\
\hline Agent & Feature & $E_{1}$ stops & $\left\{E_{1}, E_{2}\right\}$ & $\begin{array}{c}\operatorname{canSee}\left(M^{*}\right)= \\
\left\{E_{1}\right\}\end{array}$ & $\left\{E_{2}\right\}$ \\
\hline$E_{1}$ & $\begin{array}{c}\text { Attack } \\
\text { Detection } \\
\text { Messages } \\
\text { Result }\end{array}$ & $\begin{array}{c}\text { Classical } \\
\text { none } \\
M_{\text {fake }} \\
\text { full failure }\end{array}$ & $\begin{array}{c}\text { Classical } \\
\text { (post } \exists \text { ) uncertainty } \\
M^{+} \\
\text {uncertainty }\end{array}$ & $\begin{array}{c}\text { Classical } \\
\text { (post } \exists \text { ) uncertainty } \\
M^{+} \\
\text {uncertainty }\end{array}$ & $\begin{array}{c}\text { Classical } \\
\text { none } \\
M_{\text {fake }} \\
\text { full failure }\end{array}$ \\
\hline$E_{2}$ & $\begin{array}{c}\text { Attack } \\
\text { Detection } \\
\text { Messages } \\
\text { Result } \\
\end{array}$ & $\begin{array}{c}\text { Strong } \\
\text { none }(\mathrm{c}) \\
M ! \\
\text { dominance }\end{array}$ & $\begin{array}{c}\text { Strong } \\
\text { none }(\mathrm{c}) \\
M ! \\
\text { success } \\
\end{array}$ & $\begin{array}{c}\text { Strong } \\
\text { none (c) } \\
\text { none } \\
\text { failure } \\
\end{array}$ & $\begin{array}{c}\text { Strong } \\
\text { none }(\mathrm{c}) \\
M ! \\
\text { dominance } \\
\end{array}$ \\
\hline A & Result & $\begin{array}{l}\text { failure } \\
\end{array}$ & failure & failure & $\begin{array}{c}\text { failure } \\
\end{array}$ \\
\hline$\overline{G_{E_{1}}}$ & $\begin{array}{l}\text { Detection } \\
\text { Security }\end{array}$ & $\begin{array}{c}\text { none } \\
\text { compromised }\end{array}$ & $\begin{array}{c}\text { post }(\exists) \\
\text { compromised }\end{array}$ & $\begin{array}{l}\text { post }(\exists) \\
\text { restored }\end{array}$ & $\begin{array}{c}\text { none } \\
\text { compromised }\end{array}$ \\
\hline$G_{E_{2}}$ & $\begin{array}{c}\text { Detection } \\
\text { Security }\end{array}$ & $\begin{array}{l}\text { none }(\mathrm{c}) \\
\text { restored }\end{array}$ & $\begin{array}{c}\text { none (c) } \\
\text { uncertain } E\end{array}$ & $\begin{array}{c}\text { none (c) } \\
\text { uncertain } E\end{array}$ & $\begin{array}{l}\text { none }(\mathrm{c}) \\
\text { restored }\end{array}$ \\
\hline
\end{tabular}

Table 15: Overall SRA3P results, detailed view of case 5: $E_{2}$ knows $E_{1}$ as dishonest. 


\begin{tabular}{|c|c|c|c|c|c|}
\hline \multicolumn{6}{|c|}{ Case 6A: $E_{1}$ starts the attack } \\
\hline & & Case & B: $E_{2}$ starts the atta & & \\
\hline Agent & Feature & $E_{1}$ stops & & $\begin{array}{r}\operatorname{canSee}\left(M^{*}\right)= \\
\left\{E_{1}\right\}\end{array}$ & $\left\{F_{2}\right\}$ \\
\hline$E_{1}$ & $\begin{array}{c}\text { Attack } \\
\text { Detection } \\
\text { Messages } \\
\text { Result }\end{array}$ & $\begin{array}{c}\text { Classical } \\
\text { none } \\
M_{\text {fake }} \\
\text { full failure }\end{array}$ & $\begin{array}{c}\text { Classical } \\
\text { (post } \exists \text { ) uncertainty } \\
M^{+} \\
\text {uncertainty }\end{array}$ & $\begin{array}{c}\text { Classical } \\
\text { (post } \exists \text { ) uncertainty } \\
M^{+} \\
\text {uncertainty }\end{array}$ & $\begin{array}{c}\text { Classical } \\
\text { none } \\
M_{\text {fake }} \\
\text { full failure }\end{array}$ \\
\hline$E_{2}$ & $\begin{array}{c}\text { Attack } \\
\text { Detection } \\
\text { Messages } \\
\text { Result }\end{array}$ & $\begin{array}{c}\text { Strong } \\
\text { (in) label } \\
M ! \\
\text { dominance }\end{array}$ & $\begin{array}{c}\text { Strong } \\
\text { (in) label } \\
M ! \\
\text { success }\end{array}$ & $\begin{array}{l}\text { Strong } \\
\text { (in) label } \\
\text { none } \\
\text { failure }\end{array}$ & $\begin{array}{c}\text { Strong } \\
\text { (in) label } \\
M ! \\
\text { dominance }\end{array}$ \\
\hline $\bar{A}$ & Result & failure & failure & failure & failure \\
\hline$G_{E_{1}}$ & $\begin{array}{c}\text { Detection } \\
\text { Security }\end{array}$ & $\begin{array}{c}\text { none } \\
\text { compromised }\end{array}$ & $\begin{array}{c}\text { post }(\exists) \\
\text { compromised }\end{array}$ & $\begin{array}{l}\text { post }(\exists) \\
\text { restored }\end{array}$ & $\begin{array}{c}\text { none } \\
\text { compromised }\end{array}$ \\
\hline$G_{E_{2}}$ & $\begin{array}{c}\text { Detection } \\
\text { Security }\end{array}$ & $\begin{array}{l}\text { (in) label } \\
\text { restored }\end{array}$ & $\begin{array}{l}\text { (in) label } \\
\text { uncertain } E\end{array}$ & $\begin{array}{l}\text { (in) label } \\
\text { uncertain } E\end{array}$ & $\begin{array}{l}\text { (in) label } \\
\text { restored }\end{array}$ \\
\hline
\end{tabular}

Table 16: Overall SRA3P results, detailed view of case 6: $E_{2}$ knows $E_{1}$ but has not yet established a belief on $E_{1}$ 's honesty. 